\title{
Index for Volume 104
}

AUTHOR AND SUBJECT INDEX. Page numbers of errata are in italic.

Abad, J., S3.65

Abad, J. A., S2.1, S3.6

Abad, Z. G., S3.1, S3.14, S3.88

Abatzoglou, J. T., 683

Abawi, G. S., S1.1

Abbas, H. K., S3.1, S3.128

Abbasi, P. A., 403, S3.1

Abbott, C., 422

Abdo, Z., S3.86, S3.114

Abdullah, S., S3.2, S3.5, S3.165

Abello, J. F., 436

Abernethy, T., S2.1, S3.6

Abeysekara, N., S3.166

Aboughanem-Sabanadzovic, N., S3.2, S3.101, S3.106, S3.113

Abou-Mansour, E., 1021

Abraham, N. D., S3.2

Abscisic acid-cytokinin antagonism: modulation of Pseudomonas syringae resistance in tobacco, 1283

3-Acetyl-deoxynivalenol (3-ADON), 513

15-Acetyl-deoxynivalenol (15-ADON), 513

Acevedo, M., S3.2, S3.41, S3.60, S3.171

Acharya, B., S3.165

Acharya, J., S3.2

Acharya, K., S3.3

Acidovorax citrulli: analysis of lineages and type

III secretion effector genes, 1152

AĆimović, S. G., S3.3

Acosta, Y. C., S3.3

Adam, L., S3.9, S3.138

Adaskaveg, J. E., S3.3, S3.16

Adekunle, O. K., S3.4

Adhikari, B., S3.89

Adkison, H., S2.10, S3.119

Aflatoxin: contamination in Kenyan maize mills, 1221; sugarcane contamination, Texas, 462

Afroz, M., S3.4

Agarkova, I. V., S3.66

Agudelo, P., S3.107

Aguiar, J. T., S3.72, S3.109

Aguilar, C., S3.4

Aguilar-Moreno, S., S3.34

Ahern, S. J., S3.140

Ahmad, A., S3.2

Ahmad, A. A., S3.4

Ahmad, H. R., S3.9

Ahmad, S., S3.156

Ahmadpour, A., S3.4

Ahmed, H. U., S3.42, S3.53

Ahmed, Y. M., S3.37

Ahonsi, M., S3.45

Ailanthus altissima: pathogenicity and virulence

of Verticillium nonalfalfae, 282, cover photo: March

Ailloud, F., S3.72, S3.172

Ajayi, O., S3.4

Akbassova, A., S3.5

Akello, J., S3.10, S3.57

Alabi, O. J., 548, S3.5

Alam, M. M., S3.85

Ala-Poikela, M., S3.151

Alayi, T. D., 1021

Albu, S., S2.1, S3.5

Albuquerque, G. M. R., 1175

Alderman, S., S3.40

Alderman, S. C., S3.181

Alexander, M., S3.120

Alexander, R., S3.75
Alfalfa mosaic virus: variability and genetic structure in Spain, 1241

Alfaro-Fernández, A., 804

Ali, A., S2.1

Ali, S., S3.2, S3.5, S3.165

Alikulov, Z., S3.10, S3.11

Aljawasim, B. D., S2.1

Allan, E., S3.5

Allen, C., S3.5, S3.72, S3.119, S3.120, S3.168, S3.177

Allen, C. M., S3.172

Allen, H. K., S3.154

Allen, J., S3.77

Allen, T., S3.73

Allen, T. W., S2.9, S2.11, S3.1, S3.2, S3.19, S3.113, S3.122, S3.128, S3.129

Alleyne, A. T., S3.6

Allium cepa: transmission of center rot by onion thrips, 812

Allsopp, E., 1063

Al-Maghrabi, K., S3.57

Almeida, J., S3.6

Almeida, R. P. P., 27, 120

Almeyda, C. V., S2.1, S3.6

Al Rwahnih, M., 548

Al-Saleh, M. A., S3.6, S3.53

Alternaria solani: SDHI fungicide resistance, molecular characterization, 40

Altier, N., S3.6

Altowairish, M. S., S3.14

Alvarez, E., S3.7

Alvarez, R. A., S3.94

Alvis, D. N., S3.68

Amanov, O., S3.133

Amaradasa, B. S., S3.7

Amarasinghe, C. C., S3.7

Amatulli, M. T., S3.140

Amé, V., 1340

Ameen, G., S3.165

Ames, K. A., S3.15, S3.78, S3.129, S3.173

Amiri, A., 396, S3.8

Amirsadeghi, S., S3.31

Amistar Xtra, 933

Amitzur, A., S3.8

Ammar, K., 1322

Amorim, L., 769

Ampelopsis spp.: geographic distribution of

cryptic species of Plasmopara viticola, eastern

North America, 692

Amuge, T., S3.132

Amundsen, K., S3.7

Analytical and theoretical plant pathology: evaluating category scales, 575; problem of fungicide resistance with high-risk fungicides in fungicide mixtures, 324; Verticillium wilt of lettuce, model for multiseasonal spread of, 908

Anchieta, A., 1349, S3.61

Ancona, V., S3.24, S3.67

Anderson, B., S3.159

Anderson, G., S3.94

Anderson, G. M., S3.64

Anderson, J., S3.8

Anderson, J. A., 865, S2.4

Andhikari, A., S3.8

Andrade, M., S3.8

Andrade-Piedra, J., S3.51, S3.118

Ane, J. M., S3.122, S3.165

Angel, C. A., S3.3, S3.21
Angel, F., S3.21

Angel, J. C., S3.3, S3.21

Anh, V. L., 634

Anjum, R., S3.9

Antilla, L., S3.54

Ant vectors: of grapevine trunk disease pathogens, 1063

Ao, S., S3.102

Aphanomyces euteiches: real-time PCR assay for, 1138

Apium graveolens: association of 'Candidatus

Liberibacter solanacearum' with a vegetative

disorder of, 804, cover photo: August

Appel, D., S2.6, S2.7

Appressoria: melanin-independent accumulation of turgor pressure in Phakopsora pachyrhizi, 977

Arabiat, S., 40

Arabiat, S. I., S3.9

Arabidopsis thaliana: Meloidogyne chitwoodi resistance conferred by RNA interference of effector gene Mc16D10L, 1098; nondestructive imaging of nematodes in planta, 497

Arachis hypogaea: lipoxygenase activation in seed cultivars in response to Aspergillus parasiticus colonization, 1340; Tomato spotted wilt virus resistance, impact of, 202

Aram, K., S3.9

Araujo, L., 820, S3.9, S3.14, S3.99

Arcibal, E., S3.166

Arfaoui, A., S3.9

Arias, R., S3.75

Aricapa, G., S3.93

Aricapa, M. G., 436

Arif, M., S3.9, S3.21, S3.34

Arismendi, N. L., S3.83

Armengol, J., 650

Arnao, E., S3.10

Aroca, T. G., S3.109

Arseneault, T., 926

Arthropod vectors: of grapevine trunk disease pathogens, 1063, cover photo: October

Arul, J., S3.63

Arvier, R., S3.105

Aryal, S., S3.10

Asalf, B., 954

Asano, H., 634

Ashfaq, M., S3.107

Ashrafi, H., 479

Asis, R., 1340

Askora, A., S3.4

Asparagus decline syndrome, 1001

Asparagus officinalis: Fusarium crown and root rot, soil organic amendments and, 403; pollen transmission of Asparagus virus 2 and mixed infections in asparagus plants, 1001

Asparagus virus 2: pollen transmission and mixed infections in asparagus plants, 1001

Aspergillus spp.

-A. flavus: sexual reproduction in sclerotia in corn, 75 , cover photo: January

-A. parasiticus: associated with sugarcane,

Texas, 462; lipoxygenase activation in peanut seed cultivars in response to colonization by, 1340

-A. section Flavi: global transport and host association within, 462

AsSdh genes: mutations associated with SDHI resistance in Alternaria solani, 40 
Assigbetse, K., S3.30

Atallah, Z. K., 641, 779

Atehnkeng, J., S3.10, S3.139

Atkins, S., S3.41

Atkinson, S. L., S3.52

atp 9 gene: multiplex assay for detection in

Phytophthora, 733

Aucique Perez, C. E., 143

Audy, P., S3.17

Augusto, J., S3.10

Avci, U., 812

Avelar, A. S., S3.10

Avelino, J., 275

Avena sativa: modeling effects of environmental conditions on Fusarium HT2 and T2 toxin accumulation in, 57

Avila, R. T., S3.14

Avis, T. J., 1036

AVR-Pital gene: effectiveness and durability in China, 762

Babadoost, M., S3.10, S3.70

Babenko, O. N., S3.10, S3.11

Babu, B., S3.11

Baby, S., S3.37

Baccari, C., 27

Bacillus subtilis strain CU12: factors affecting plant pathogen sensitivity to fengycin, 1036

Backman, P., S3.161

Backman, P. A., 379

Bacon, C. W., S2.7

Bacteriology: abscisic acid-cytokinin modulation of Pseudomonas syringae resistance in tobacco, 1283; Acidovorax citrulli lineages and type III secretion effector genes, 1152; 'Candidatus Liberibacter asiaticus', effect of citrus juice on viability in culture medium, 15; 'Candidatus Liberibacter solanacearum', association with a vegetative disorder of celery, Spain, 804; 'Candidatus Liberibacter solanacearum', physiological responses of potato cultivars to, 126; colonization of dormant walnut buds by Xanthomonas arboricola pv. juglandis, 1163; comparative analysis of Burkholderia glumae and B. gladiolli, 436; definition of plantpathogenic genomospecies in the Pseudomonas syringae complex, 1274; Moko disease-causing strains of Ralstonia solanaceraum phylotype II in Brazil, 1175; Ralstonia solanacearum, diversity in French Guiana, 586; roles of solo LuxR in Lysobacter enzymogenes strain $\mathrm{OH} 11$, 224; transmission of causal agents of center rot of onion by onion thrips, 812 ; Xanthomonas citri subsp. citri control, foliar application of biofilm formation-inhibiting compounds, 134; Xanthomonas euvesicatoria, role of blossom colonization in pepper seed infestation, 232; Xanthomonas oryzae pv. oryzae, genetic diveristy of transcriptional activator-like effector genes, China, 672; Xylella fastidiosa, blocked diffusible signal factor and insect transmission, 27; Xylella fastidiosa, genetic structure in sweet orange, 120

Badillo-Vargas, I. E., S3.158

Bag, S., S3.107

Bai, G.-H., 472

Bailey, B., S3.67, S3.184

Baird, R. E., S3.1

Baird, S. M., S3.73, S3.74

Baker, H. N., S3.162, S3.163

Baker, K., S3.113

Bakkeren, G., S1.3

Balatti, P. A., 357, S3.11

Balbalian, C., S3.122

Balci, Y., S3.1
Baldauf, C., 769

Balk, C., S3.166

Ballen, C., 436

Bandara, A. Y., S3.11

Bandyopadhyay, R., S3.10, S3.54, S3.57, S3.139

Banerjee, N., S3.110

Bano, A., 933

Banuelos, G., S3.124

Bao, Y., S3.25

Barak, J. D., S3.112

Barandoc-Alviar, K., S3.11

Barba, P., S3.152

Barbeau, A. D., S3.175

Barclay, S., S3.103

Bardin, M., 859

Bardsley, S., S3.12

Bargeron, C. T., S3.27

Baric, S., S3.105

Barker, G., S3.54

Barman, A. K., 812

Barnhill, J., S3.85

Barocco, R. L., S2.3

Barphagha, I. K., S3.87

Barranco, D., S3.120

Barros, M. M., S3.47

Bartels, D. W., S3.64

Barton, W., S3.42

Bartz, J. A., S3.113

Basallote-Ureba, M. J., 403

Basidiomycetes: associated with wood rot of citrus, Italy, 851

Basu, U., S3.138

Batche, H., S3.179

Bateman, C., S3.147

Bateman, M., S3.93

Battaglia, E., S3.154

Baudoin, A., 692, S3.160

Baum, T. J., 879

Baumgartner, K., S3.12, S3.43, S3.67

Baur, M., S3.181

Bayala, R., S3.30

Bayles, M. B., 1088

Bayles, R., 871

Baysal-Gurel, F., S3.12, S3.142

Bean common mosaic necrosis virus:

recombinant, over coming resistance in common bean, 786; recombinant, temperature-insensitive necrosis in common bean, 1251

Bean common mosaic virus: on common bean, cover photo: November; interaction of genetic determinants with common bean resistance

factors, 786; temperature-insensitive necrosis in I gene-bearing common bean, 1251

Bean pod mottle virus: geospatial and temporal analysis of epidemics in soybean, 365

Beasley, E. D., S3.103

Beattie, A. D., S3.120

Beattie, G., S3.166

Beattie, G. A., S3.71, S3.81, S3.173

Beattie, M. S., S3.29

Beaubrun, J. J., S3.55

Beaudoin, E., S3.123

Beaudry, R. M., S3.50

Beaulieu, C., S3.65

Beaver, J. A., S3.90

Becker, M. G., S3.12, S3.46, S3.135

Beckerman, J., S3.159

Becktell, M. C., S3.12

Bederski, K., S3.133

Beed, F., S3.51

Beenen, H., S3.154

Behrens, T., S3.127

Beirn, L., S3.13

Beirn, L. A., S1.1, S3.160

Bekoscke, K., S3.13
Bélanger, R. R., S1.3

Belasque, J., S3.18, S3.86

Bellaloui, N., S3.1

Belmar, S., S2.11, S3.124

Belmonte, M. F., S3.12, S3.46, S3.74, S3.135

Bender, B., S3.166

Benedict, C., S3.181

Bennett, J. W., S3.67

Benson, D. M., S3.17, S3.44, S3.146, S3.147

Bentancur, O., 715

Benzothiadiazole: effect on development of gray leaf spot in perennial ryegrass, 614; gray mold resistance induced in tomato, 150

Berbegal, M., 650

Berendsen, S., S3.68

Beresford, R. M., 50

Berg, J., S3.76, S3.182

Bergey, B., 660

Bergstrom, G. C., 513

Bergua, M., 1241

Berguson, W., S3.36

Berkett, L. P., S1.5

Berner, D., S3.160

Berner, D. K., S3.161

Bernert, A., S3.13, S3.103

Bertaccini, A., S3.64

Bertolini, E., 804

Bertsch, E., 1021

Besler, K., S3.13

Beta vulgaris: coupling spore traps and $\mathrm{qPCR}$ assays to detect downy mildew of, 1349; Fusarium oxysporum isolates, multilocus analysis, 886

$B$ genes: pyramiding, bacterial blight resistance in cotton and, 1088

Bhatnagar, D., S3.84, S3.95

Bhavani, S., S3.19

Bhowmick, T. S., S3.140

Bhuiyan, M. A. H., S3.13

Bian, Z., S3.13

Bianco, T., S3.125

Bienapfl, J. C., S3.1, S3.14, S3.88

Bietila, E., S3.14

Bignell, D. R., S3.14, S3.25

Bilgri, A., S3.14

Bilodeau, G. J., 733, S3.14, S3.80, S3.98

Bilton, Z. O., S3.87

Biochemistry and cell biology: carbohydrate metabolism changes in Prunus persica infected with Lasiodiplodia therobromae, 445; Ceratocystis fimbriata resisitance in mango, 820; differential responses of grapevine cultivars to Botryosphaeria dieback, 1021; effect of membrane lipids on plant sensitivity to fengycin, 1036; effect of silicon on soybean resistance to Cercospora sojina, 1183; insertional mutagenesis and cloning of the gene controlling M5HF2C toxin in Cochliobolus lunatus, 332; Lilium LsGRP1 protein dual subcellular localization, 1012; Pyricularia oryzae, effects on leaf gas exchange and chlorophyll $a$ fluorescence in silicon inoculated wheat, 143; Rhizopus oryzae, increasing spore susceptibility to heat stress, 240; silicon-potentiated phenylpropanoid pathway in banana roots during Fusarium oxysporum f. sp. cubense infection, 597

Bioflim formation-inhibiting compounds: foliar application to control Xanthomonas citri subsp. citri, 134

Biological control: anatagonistic interactions of 'Candidatus Phytoplasma mali' strains, 453, cover photo: May; Cryphonectria hypovirus infection, dynamics in chestnut blight cankers, 
918; induction of priming-mediated systemic resistance in cucumber against Colletotrichum orbiculare, 834; infection route of a Fusarium seed pathogen into Bromus tectorum seeds, 1306; long-term induction of defense gene expression in potato, 926; multiantibiotic complex produced by Streptomyces araujoniae against Botrytis cinerea, 1298, cover photo:

December; potential of Pseudomonas chlororaphis subsp. aurantiaca strain Pcho10 against Fusarium graminearum, 1289; roles of solo LuxR in Lysobacter enzymogenes strain $\mathrm{OH} 11,224$; soil fumigation and Clonostachys rosea against cucumber wilt, 1314; of wheat root diseases by Pseudomonas fluorescens HC1-07, 248

Biondi, E., S3.64

Bipolaris sorokiniana: histochemical

characterization of early response to infection in barley, 715

Birr, T., S3.61, S3.122

Bischoff, J. F., S3.146

Bishop, K. D., S3.55

Bispo, W. M. S., 820, S3.9, S3.14, S3.99

Bissonnette, K. M., S3.15

Bitsadze, N., S3.15

Bittner, R., S3.15

Blakeslee, J., S3.63

Blanchette, R. A., S3.51

Bledsoe, M., S3.144

Bloechle, T. M., S3.166

Blomquist, C. L., 733

Blonde, G., S3.111

Bloome, G., S3.51

Bloomingdale, C., S3.167

Blosberg, J., S3.103

Bluhm, B., S3.39, S3.153

Bluhm, B. H., S3.10, S3.95

Blumeria graminis f. sp. tritici: on wheat,

trehalose induction of wheat defenses, 293

Bo, W., S3.147

Boal, R. J., S3.132

Bock, C. H., 575, S3.15, S3.16, S3.18, S3.26, S3.37, S3.86

Bockus, W., 472

Bocsanczy, A. M., S3.16

Boenisch, M. J., S3.134

Boer, R., S3.24

Bogdanove, A., S3.120

Bohannon, S., S3.136

Bolton, M. D., 40, S3.13, S3.16, S3.17, S3.36

Bomberger, R. A., S3.17, S3.125

Bond, J., S3.53, S3.127

Bonde, M. R., S3.17, S3.89

Bonello, P., S3.63, S3.107, S3.148

Bonhoeffer, S., 324

Bonierbale, M., 624

Bonkoungou, S. E., S3.139

Booker, H., S3.36

Boosted regression trees: predicting Fusarium

head blight epidemics, 702

Booth, C., 232

Borneman, J., S3.133

Bornemann, K., S3.17

Bornowski, N., S3.169

Borrego-Benjumea, A., 403

Borron, S., S3.98

Borth, W., S3.32, S3.79, S3.162

bosl gene, 396

Boscalid resistance: in Alternaria solani,

molecular characterization, 40; spatial

distribution of SNPs related to, 604

Bostic, L. E., S3.17

Bostock, R. M., S3.77, S3.99

Botero, D., S3.97
Botryosphaeria dieback: differential responses of grapevine cultivars to, 1021

Botrytis spp.

- B. cinerea: biocontrol with Streptomyces araujoniae, 1298, cover photo: December; fitness and competitive ability of isolates with dual resistance to SDHI and QoI fungicides, 347; fludioxonil resistance in isolates from blackberry and strawberry, 724; genotypes in tomato greenhouse populations, 859 ; iprodione resistance in isolates from strawberry and blackberry, 396; spatial distribution of SNPs related to fungicide resistance, 604

- B. cinerea resistance: induced in tomato by benzothiadiazole and Trichoderma harzianumi T39, 150

-B. elliptica: LsGRP1 protein expression profile in Lilium following infection, 1012

-B. squamosa: spatial distribution of SNPs related to fungicide resistance, 604

Boucher, C., S3.53

Bourdon, N., 293

Bowden, S., 871

Bowen, C. R., S3.170

Bowen, K., S3.17, S3.104

Bowen, K. L., S3.49, S3.68

Bowman, J. E., S3.157

Bowman, M. J., S3.130

Boyetchko, S. M., S3.17

Boysenberry: downy mildew disease risk prediction model, 50. See also Rubus spp.

Boyzo-Marin, J., S3.18

BP15 synthetic antimicrobial peptide, 1192

BP22 synthetic antimicrobial peptide, 1192

BP25 synthetic antimicrobial peptide, 1192

Bradley, C., S3.10

Bradley, C. A., S2.3, S3.4, S3.15, S3.28, S3.38, S3.58, S3.78, S3.129, S3.135, S3.173

Bradley, M., S3.84

Bradshaw, R. E., S3.154

Bradshaw, T. L., S1.5

Braido, R., S3.18

Brake, A., S2.2

Brand, S. C., S3.18

Brannen, P. M., S3.16, S3.143, S3.183

Brantner, J. R., S3.18

Brar, G., S3.18

Brassica napus: effects of cropping on the genetic structure of Plasmodiophora brassicae, 532; role of primary and secondary infection in plant response to Plasmodiophora brassicae, 1078

Braswell, E., S3.102, S3.121

Breakspear, A., S3.48

Brellenthin, N., S3.83

Brenneman, T., S2.2, S3.19

Brenneman, T. B., S2.10, S3.155

Bressan, M., 1138

Breunig, M., S3.19

Brewer, M., S3.16, S3.68

Brewer, M. T., S3.153

Briar, S., S3.117

Brick, M. A., S3.99

Briggs, J., S3.19

Bright, D. B., S3.47, S3.131

Brito, R. A. S., S3.19, S3.23

Brlansky, R. H., S3.101

Broccardo, C. J., S3.128

Brochard, N., S3.19

Broderick, K. C., S3.167, S3.174

Broders, G., S1.1, S3.123

Broders, K., S3.46, S3.69, S3.123, S3.124

Broders, K. D., S1.1, S3.135

Brodeur, L., 604

Bromus tectorum: infection route of a Fusarium seed pathogen into the seeds of, 1306
Brown, C., S3.103, S3.106

Brown, C. R., 1098, S3.37

Brown, D. W., S3.140, S3.170

Brown, E., S3.55, S3.87

Brown, E. W., S3.98

Brown, J. K., S3.6, S3.10, S3.20, S3.26, S3.40, S3.53, S3.179

Brown, K., S3.19

Brown, P. J., S3.24

Brown, R., S3.84

Brown, R. L., S3.95

Browne, G., S3.114

Browne, G. T., S3.20, S3.104

Browne, P., S3.144

Brown Horowitz, S., 484

Browning, K., S3.94

Browning, K. S., S3.174, S3.177

Brown planthopper, 196

Broz, K. L., S3.20, S3.134

Bruce, A., S3.20, S3.108

Bruce, J. E., S3.141

Bruckart, W. L., S3.160

Bruckart, W. L. III, S3.161

Bruckner, P., S3.76, S3.182

Brueggeman, R., S3.54

Bruening, S. R., 1107

Brunings, A., S3.91

Bruns, T., S3.20

Brurberg, M. B., S3.115

Bryant, R., 871

Bryner, S. F., 918

Buchner, R., 1163

Bucker Moraes, W., S3.20

Buckler, E., S3.153

Buckner, S., S3.117

Buell, R., S3.169

Bunkers, G. J., S3.21

Burbank, L., S3.21, S3.130

Burciaga, R., S3.60

Burdman, S., 1152

Burgess, T., S3.1

Burkey, K. O., S3.75

Burkholderia spp.

-B. gladioli: comparative analysis, 436

-B. glumae: comparative analysis, 436

Burns, D., S2.5

Burns, K. S., 163

Burrell, D., S2.9

Burris, G., S2.5

Burrows, E., S3.87

Burrows, M., S3.183

Burrows, M. E., S3.94

Bush, E., S3.76

Bushoven, J., S3.27

Bushula, V. S., S3.167

Busman, M., S3.140

Busot, G., S3.9, S3.21

Butler, A. M., S3.1

Butler, D., S3.52

Butler, D. M., S3.108

Byamukama, E., 365, S3.3, S3.116

Cacao agroforests: frosty pod rot, effects of shade tree spatial structure on, 275

Cacciola, S. O., 851

Cacique, I. S., 820, S3.9, S3.99

Cadavid, M., S3.3, S3.21

Cadle-Davidson, L., S3.152

Caglayan, K., 108

Cai, M., S3.21

Caicedo, A., S3.70, S3.71

Cale, J. A., S1.2, S3.158

Callicott, K. A., S3.54, S3.139

Calotropis procera: leaf extracts, efficacy against wheat leaf rust, 933 
Cambra, M., 804

Campbell, A., S3.148

Campbell, A. S., S3.21

Cañas-Duarte, S., S3.97

Canavan, S., S3.33

'Candidatus Liberibacter asiaticus': acquisition efficiency by Diaphorina citri and temporal progression of infection, 416; detection in citrus and Diaphorina citri, Pakistan, 257; effect of citrus juice on viability in culture medium, 15; population structures, China, 158

'Candidatus Liberibacter solanacearum': association with a vegetative disorder of celery, Spain, 804, cover photo: August; physiological responses of potato cultivars to, 126 ; real-time PCR assay, 804

'Candidatus Phytoplasma mali': antagonistic interactions of strains in Catharanthus roseus and Nicotiana occidentalis, 453, cover photo: May

Candresse, T., 660

Cannesan, M.-A., 1138

Cantu, D., S3.95

Cao, T., S3.22

Capouya, R. D., S3.68

Capretti, P., S3.107

Capsicum annuum: Cucumber mosaic virus infection, molecular genetic analysis of populations in Korea, 993; Phytophthora fruit rot resistance, mapping, 479; Xanthomonas euvesicatoria seed infestation, role of blossom colonization in, 232

Carbone, I., 75, S3.68, S3.118

Cardenas, M., S3.97

Cardona, M. B. B., S3.99

Cardoza, Y. F., S3.22

Cardozo, C., S3.3

Cardwell, K. F., S3.157

Carisse, O., 604, 692, S3.22, S3.65

Carlson, J., S3.143

Carmeli, S., 240

Carrari, V., 1340

Carris, L. M., S3.77

Carroll, B., S3.144

Carroll, M., S3.143

Carta, L. K., S3.22

Carvajal-Arias, C. E., S3.99

Carvajal Yepes, M., S3.51, S3.118

Carvalho, C. M., S3.22, S3.112

Carvalho, S. A., S3.18, S3.86

Carvalho, S. L., S3.22

Cary, D., S3.3

Casela, C. R., S3.66

Cassone, B. J., 1360

Castagnora, A. P., 970

Castanea spp.: dynamics of Cryphonectria

hypovirus 1 infection in chestnut blight cankers, 918

Castel, L., 1138

Castell-Miller, C., S3.4, S3.23, S3.40

Castello, J. D., S1.2, S3.158

Castiblanco, L. F., S3.23

Castiglioni, C., 1201

Castillo, K. D., S3.179

Castlebury, L., S1.2, S3.77

Castlebury, L. A., S3.31

Castroagudin, V. L., 95, S3.23

Caswell-Chen, E. P., S3.129

Category scales: advantages and disadvantages, 575

Catharanthus roseus: antagonistic interactions of

'Candidatus Phytoplasma mali' strains in, 453, cover photo: May

Cavalcante, G. P., S3.19, S3.23

Cavin, C. A., S3.160, S3.161

Ceja-Torres, L. F., S3.18, S3.24
Celebi-Toprak, F., 211

Celik Oğuz, A., S3.23

Cellier, G., 1175

Cell wall appositions: in barley response to

Cochliobolus sativus infection, 715

Centofanti, T., S3.124

Ceratocystis fimbriata: responses of mango to infection, 820

Cercospora spp.

- C. kikuchii: TaqMan-qPCR assay for detecting

in soybean leaves, 1118

-C. sojina resistance: in soybean, effect of silicon on, 1183

Ceresini, P. C., 95, S3.23, S3.24

Cha, B.-J., 993

Chagas Silva, E., S2.2

Chain, P. S., S3.125

Chan, C. H., S3.136

Chanda, A., S2.6

Chanda, A. K., 1118, S2.2, S3.109, S3.154

Chang, C. J., S3.115

Chang, H. X., S3.24

Chang, H.-X., 977

Chang, J., S3.24

Chang, K. F., S3.53

Chang, M.-W., 340

Chang, S., S3.24

Chang, T., S1.2

Chao, S., S3.71

Chapados, J. T., 422

Chapara, V., S3.167

Chapuis-Lardy, L., S3.30

Charkowski, A., S3.5, S3.14

Charkowski, A. O., S3.43, S3.45, S3.101, S3.169, S3.170, S3.171

Chastagner, G. A., S3.180

Chatnaparat, T., S3.24

Chatterton, S., S3.96

Chauhan, R. P., S2.2

Chavarro Mesa, E., S3.24

Chavez, J. D., S3.141

Chavez, S., S3.63, S3.121

Chawner, M., S3.167

Cheesman, H. K., S3.161

Chen, C., S1.4, S3.16

Chen, C. Y., S3.113

Chen, C.-Y., 340, 1012

Chen, G.-Y., 672

Chen, H., S3.25

Chen, J., 158, 332, S3.27, S3.115, S3.137

Chen, L., 269

Chen, P., S3.69, S3.121

Chen, R., S3.50

Chen, S., S3.25, S3.119, S3.132

Chen, W., S3.60

Chen, X., S3.39, S3.125

Chen, X. M., 1208

Chen, Y., 1289, S3.25, S3.132, S3.168

Chen, Y. H., S3.25

Chen, Z. Y., S3.95, S3.97, S3.154

Chen, Z.-Y., 1118

Cheng, J., S3.89

Cheng, P., 1208, S3.26

Cheng, Q., S3.158, S3.164

Cheng, Z., S3.25, S3.96

Chenopodium quinoa: Peronospora variabilis, molecular detection and phylogeny, 379; seed heads, cover photo: April

Cheon, W., S3.26

Cheung, H. K., S3.26, S3.35

Chiang, K.-S., 575, S3.15

Chiang, K. S. P., S3.26

Chiesa, M. A., 970

Chikh-Ali, M., S3.180

Childers, R. A., S3.30
Childs, K. L., S3.130

Chilvers, M., S2.10, S3.40, S3.58, S3.100, S3.127

Chilvers, M. I., S3.176

Chingandu, N., S3.26

Chitarra, W., S3.48

Chitrampalam, P., S3.2, S3.27

Chittem, K., S3.54

Chitwood, D. J., S3.172

Chlorophyll $a$ fluorescence: effects of silicon on

in Pyricularia oryzae infected wheat, 143

Cho, H., S3.44

Cho, S. Y., S3.27

Choi, H.-S., 993

Choi, O., S3.64

Choi, Y. J., S3.61

Choi, Y.-J., 1349

Chong, J., 1021

Choo, H. S., S3.17

Choudhury, R. A., S3.180

Chowdhury, R., S3.116

Christensen, S., S3.139

Christianson, C. E., S3.180

Chu, Y., S2.7

Chuma, I., 634

Chung, B., S3.81

Chung, B. K., S3.64

Ciampi-Guillardi, M., 769

Cilas, C., 275

Cilia, M. L., S3.141

Cilindre, C., 1021

Citrus greening disease. See 'Candidatus

Liberibacter asiaticus'

Citrus juice: effect on 'Candidatus Liberibacter asiaticus' viability in culture medium, 15

Citrus spp.: 'Candidatus Liberibacter asiaticus', detection, Pakistan, 257; 'Candidatus Liberibacter asiaticus' infection, temporal progression and acquisition efficiency by Diaphorina citri, 416; characterization of basidiomycetes associated with wood rot, Italy, 851; Colletotrichum acutatum populations, genetic structure, Brazil, 769

-C. limon: characterization of basidiomycetes associated with wood rot, Italy, 851

-C. sinensis: genetic structure of Xylella fastidiosa, 120

-C. unshiu: canker disease resistance, role of leaf features in, 970

Citrus stubborn disease: novel diagnostic method using an Spiroplasma citri-secreted protein, 188 Clark, C. A., S2.4

Clark, I. M., S3.99

Clark, N., S3.27

Clarke, B., S1.1

Clarke, B. B., S3.13, S3.160

Clarkson, J., S3.86

Class II glycine-rich proteins, 1012

CLAVATA3/ESR-related ligand proteins, 879

CLE ligand proteins, 879

Clemens, C., S3.3

Clément, C., 1021

Clement, D. L., S3.27

'Clemenules' mandarin, 970

Cleveland, K., S3.27, S3.169

Cleveland, K. M., S3.168

Clonostachys rosea: synergistic effect with soil

fumigation against cucumber wilt, 1314

Cloutier, S., S3.36

Clt-1 gene: insertional mutagenesis and cloning, 332

Coats, K., S3.180

Cober, E., S3.132

Cochliobolus spp.

-C. lunatus: insertional mutagenesis and cloning of the gene controlling M5HF2C toxin, 332 
-C. sativus: histochemical characterization of early response to infection in barley, 715

Cochran, A., S3.59

Cochran, A. M., S2.3, S3.28

Cochran, K., S2.2, S3.28

Coelho-Netto, R. A., 365

Coffey, M., S3.1

Coffey, M. D., 733, S3.80, S3.100

Coffman, R., S3.157

Cohen, C. K., S3.157

Cohen, S., S3.120

Coletta-Filho, H. D., 120, 416

Collemare, J., S3.154

Colletotrichum spp.

-C. acutatum: genetic structure, Brazil, 769

-C. gloeosporioides resistance: in strawberry, genetic variation and, 67

-C. orbiculare resistance: in cucumber, induction of, 834

Colletti, A. R. S., S3.28

Collins, B. D., S3.28

Collins, S. B., S3.88

Commer, B. E., S2.3

Comstock, J. C., S3.100

Concibido, V. C., S3.28

Coniophora spp.: on citrus in Italy,

characterization of, 851

Conn, K. L., S3.17

Conner, R. L., S3.53

Conrad, A. O., S3.148

Conrad, C., S3.128

Cook, D. R., S3.122

Cooke, D. E. L., S3.37

Coombs, J., S3.169

Cooper, M., S3.12

Coram, T. E., S3.40

Coranson-Beaudu, R., 586

Corcuff, R., S3.63

Cornejo, F., S3.44

Corral, R., 520

Correa, F., 436

Corylus avellana: Cytospora canker, effect of summer heat and soil organic matter on severity, 387

Costa, R. V., S3.66, S3.112

Costa de Novaes, M. I., S3.90

Cota, L. V., S3.66, S3.112

Côté, C., S1.1

Cotton, C., S3.67

Cotty, P., S3.10

Cotty, P. J., 462, S3.54, S3.57, S3.110, S3.139

Cottyn, B., 520

Court, L. N., 749

Coutinho, B. G., S3.28

Coutinho, T., S3.36

Couture, C., 660

Covey, P., S3.128

Covey, P. A., 886

Cox, B. A., S3.29

Cox, K., S3.13, S3.29, S3.83

Cox, K. D., 945, S1.4, S3.122

Coyne, C., S3.26

$\mathrm{Cr} 4$ gene: white pine blister rust resistance and, 163

Cramer, G., S3.43

Cranmer, T. J., S3.29

Cranshaw, W., S3.48, S3.53, S3.135

Craven, J., S3.120

Craze, M., 871

Crenshaw, N., S3.46

Creswell, T., S3.81

Creswell, T. C., S3.155

Crevelin, E. J., 1298

Croce Filho, J., S3.86

Crocker, E., S3.29
Croll, D., S3.152

Cronartium ribicola resistance: in limber pine, 163

Cross, D., S3.40

Crouch, J. A., S1.1, S1.2, S1.5, S3.13, S3.74, S3.98, S3.102, S3.146, S3.147, S3.160, S3.163

Crous, P. W., S3.24

Crow, W. T., S3.10

Crump, N. S., S3.32

Cruse-Sanders, J., S3.111

Cruz, D. R., S3.29

Cryphonectria hypovirus 1: dynamics in chestnut

blight cankers, 918

Cryphonectria parasitica: infection with

Cryphonectria hypovirus 1, 918

Cuasquer, J., S3.93

Cucumber mosaic virus: molecular genetic analysis of populations infecting pepper in Korea, 993

Cucumis sativus: control of Fusarium wilt with soil fumigation and Clonostachys rosea, 1314; induction of priming-mediated systemic resistance against Colletotrichum orbiculare, 834

Cui, J., S3.126

Culbreath, A. K., 202

Cumagun, C. J. R., 634

Cummings, J. A., 513

Cummings, T. F., S3.42, S3.158

Curland, R. D., S3.74, S3.78, S3.113, S3.173

Curry, K. J., S3.111

Cyclic-lipopeptide producing Pseudomonas fluorescens HC1-07, 248

Cyst nematodes: effector proteins, 879

Cytochrome b (cyt b) gene: G143a mutation, 347;

heteroplasmy in Venturia inaequalis, and its role in trifloxystrobin resistance, 945

Cytokinin-abscisic acid antagonism: modulation of Pseudomonas syringae resistance in tobacco, 1283

Cytospora corylicola: in hazelnut, effect of summer heat and soil organic matter on severity, 387

Czemmel, S., S3.43

Daayf, F., S3.9, S3.57, S3.81, S3.138

d'Afonseca, D. S., S3.72, S3.109

Daglish, G., S3.51

da Graça, J. V., S3.63, S3.64

Dahan, J., S3.180

Daily, S., S3.131

Dale, A. L., S3.153

Dallot, S., S3.98

Dally, E. L., S3.161

Dalsing, B. L., S3.120, S3.168, S3.177

Dalton, J., S3.32

Damann, K., S3.29

DaMatta, F. M., 34, 143, 1183, S3.14, S3.99

Damicone, J., S2.3

Dandurand, L. M., S3.30, S3.85

Dangi, S., S3.69, S3.168

Dangi, S. R., S3.30

Daniels, D. A., S3.30

Danies, G., S3.30

Danilova, T., S3.60

Dao, S., 520

Dart, N., S3.147

Dart, N. L., S3.147

Das, M., S3.140

da Silva, W. L., 597

Dattamajumder, S. K., S3.110

Daugherty, M. P., 416

Daughtrey, M., S2.4

Daughtrey, M. L., S3.44, S3.147

Davies, K. A., 174
Davies, L. J., S3.37

Davis, D. D., 282, S3.148, S3.162

Davis, E. L., 879

Davis, R., S2.2

Davis, R. E., S3.161

Davis, R. F., S3.30

Davis, R. M., S3.33

Davis, V. M., S3.58

Dawood, D. H., 1289

Dawson, W. O., S3.140

DeBauw, A., S3.180

Debenport, S., S3.30

Deberdt, P., 586

DeBlasio, S. L., S3.141

De Boer, S. H., 422

Debona, D., 34, 1183

DeBuse, C., S3.40

de Cock, A. W., S3.113

Decognet, V., 859

Dedrick, M., S3.88

Dedryver, F., 1042

Dee, M. M., S3.88

Defense gene expression: long-term induction in potato, 926

Dehesh, K., S3.99

Deheuvels, O., 275

Deiulio, G., S3.31

de Kievit, T., S3.12, S3.74

de Koeyer, D., 624

De La Fuente, L., 15, S3.58

de la Garza, C., S3.63

Delaunay, A., S3.98

Del Castillo-Múnera, J., S3.31

Delfosse, P., S3.15, S3.26, S3.37

Delière, L., 692

Delmotte, F., 692

Del Ponte, E., S3.109

Del Ponte, E. M., 513

del Rio, L., S3.42, S3.138

del Rio Mendoza, L. E., S3.31, S3.74, S3.84

Demers, J., S1.2, S3.31

deMilliano, E. J., S3.138

de Mol, F., 532

De Moraes, C., S3.151

Deng, H., S3.131

Deng, P., S3.73, S3.74

Deng, W. L., S3.115

Deng, X., 158, S3.137

Dennis, G. G., S3.130

Densley, A. D., S3.31

de Olivera, C., S3.131

Deora, A., 1078, S3.29, S3.31, S3.32

Deoxynivalenol (DON): in Fusarium-infected winter wheat, 472; production by Fusarium graminearum related to aggressiveness, 357; production by Fusarium graminearum related to disease severity in soybean, 1201

Derevnina, L., S3.75

DeRobertis, C., S3.29

Derr, J., S3.76

Desaeger, J. A., S3.163

Desgagnes, R., S3.17

Detemmerman, L., 520

Determann, R., S3.111

de Vallavieille-Pope, C., 1042, S3.46

Devengenzo, M. T., S3.20, S3.104

DeVries, R. M., S3.130

Dewdney, M. M., S2.10, S3.117, S3.121, S3.126

De Wit, P. J., S3.154

De Wolf, E. D., 702, S3.47

Dey, K. K., S3.32

Dharjono, V. N., S3.32

Dhiman, C., S3.77, S3.85, S3.183

Dhingra, A., S3.89 
Di, R., S3.32

Diao, Y., S3.32, S3.83

Diaphorina citri: acquisition efficiency of 'Candidatus Liberibacter asiaticus' and the temporal progression of infection, 416; detection of 'Candidatus Liberibacter asiaticus' in, Pakistan, 257

Diaz, C., S2.3, S3.33

Díaz, E. A., S2.4

Diaz, G. A., S3.33, S3.40

Diaz, S. T., S3.184

Diaz, T., S3.43

Diaz-Lara, A., S3.33

Di Bello, P., S3.32

Dicarboximide resistance: spatial distribution of SNPs related to, 604

Dichosa, A. E., S3.125

Dick, R. P., S3.30

Diers, B. W., S3.72

Diez, C. M., S3.120

Diez de la Fuente, P., S3.41

Diffusible signal factor, 27

Dil, T., S3.23

Dillard, H. R., S3.15, S3.156

Dill-Macky, R., S3.8, S3.39, S3.113

Dimock, M., S3.51

Dimock, M. B., S3.54

Ding, S., S3.169

Dinh, P. T. Y., 497, 1098

Disease assessment scales: advantages and

disadvantages of category scales, 575

Disease control and pest management: antimicrobial trait of Lilium LsGRP1 protein, 340; fitness and competitive ability of Botrytis cinerea with dual resistance to SDHI and QoI fungicides, 347; gray mold resistance induced in tomato by benzothiadiazole and Trichoderma harzianumi T39, 150; heteroplasmy of the cytochrome $b$ gene in Venturia inaequalis and its role in trifloxystrobin resistance, 945; inhibitory effects of stilbenes on in vitro growth of soybean pathogens, 843; pathogenesis-related protein expression in wheat leaves pre-treated against leaf rust with plant extracts, 933; postinfection activity of synthetic antimicrobial peptides, 1192; Pyricularia oryzae impairment of wheat leaf photosynthesis, 34; race specificity of Puccinia striiformis resistance in wheat, 1042; Rhizoctonia, agroecological factors correlated to soil DNA concentrations, 683; SDHI fungicide resistance in Alternaria solani, molecular characterization, 40; soil fumigation and Clonostachys rosea against cucumber wilt, 1314

Disease risk prediction models: for downy mildew in boysenberry, 50

Ditylenchus dipsaci: FTA technology for DNAbased analyses, 306

D-leucine, 134

DNA-based analyses: of plant-parasitic nematodes with FTA technology, 306

Doa, M., S3.33

Doan, H., S3.33

Dobhal, S., S3.34

Dobson, A., 954

Dolovac, N. S., S3.34, S3.120

Dombroskie, J. J., S3.77

Domier, L. L., S3.24, S3.133

Donda, B. P., S3.34

Dong, Y., 513, S3.15

Dong, Y.-H., 472

Donofrio, N. M., S3.162

Dopamine: in banana roots supplied with silicon, 597, cover photo: June

Dorman, E., S3.81
Dorosky, R. J., S3.125

Dorrance, A. E., S3.68, S3.72, S3.165, S3.166

Dorrestein, P., S3.176

Douce, G. K., S3.77

Douches, D., S3.169

Douda, O., 306

Douglas, C. J., S3.65

Douglas, S. M., S3.147

Doussoulin, H. A., S3.83, S3.188

Dowell, F., 472

Doyle, C., S3.35

Doyle, V., S2.1, S3.5

Draper, M. A., S3.156

Dreaden, T., S3.35

Dreaden, T. J., S3.148

Driouich, A., 1138

Driver, J., S3.109, S3.129

D'Souza, D. H., S3.20

Du, L., 224

Duan, Y., S3.92

Duarte, V., S3.22

Dubin, H. J., S3.160

Dudley, K., S3.55

Dudley, M., S3.35

Dufault, N., S3.11

Dufault, N. S., S2.3

Dufour, D., S3.7

Dugan, F. M., S3.35, S3.60

Duitama, J., 436

Dumenyo, K., S3.35

Dung, J. K., S3.181

Dunham, J. P., S3.110

Dunn, A. R., 1107

Dunnell, K. L., S3.36

Duong, L. T., S3.36

Duque, M. C., S3.93

Duranovic, X., S3.17

Durgan, B. R., S3.157

Dutbayev, Y., S3.36, S3.133

Dutillleul, P., 604

du Toit, L. J., S3.28, S3.180

Dutta, B., 232, 812, S2.3, S2.9, S3.36, S3.75

Dyer, A., S3.76, S3.182

Earl, H. J., S3.30

Earlywine, D. T., S3.81

Earnest, J. D., S3.110

Ebert, M., S3.36

Ebskamp, M. J., S3.78

Eckshtain-Levi, N., 1152

Ecology and epidemiology: arthropod vectors of grapevine trunk disease pathogens, 1063, cover photo: October; Aspergillus parasiticus associated with sugarcane, Texas, 462; Bean pod mottle virus epidemics in soybean, geospatial and temporal analysis, 365; Botrytis cinerea, genotypes in tomato greenhouse populations, 859; 'Candidatus Liberibacter asiaticus', acquisition efficiency by Diaphorina citri and temporal progression of infection, 416; 'Candidatus Liberibacter asiaticus', detection in citrus and Diaphorina citri, Pakistan, 257;

'Candidatus Liberibacter asiaticus' population structures, China, 158; characterization of basiodiomycetes associated with wood rot of citrus, Italy, 851; disease risk prediction model for downy mildew in boysenberry, 50; frosty pod rot in cacao agroforests, effects of shade tree spatial structure on, 275; Fusarium crown and root rot of asparagus, soil organic amendments and, 403; Fusarium graminearum, toxigenic capacity and trichothecene production related to aggressiveness in wheat, 357; Fusarium head blight, predicting epidemics with boosted regression trees, 702; hazelnut cytospora canker, effect of summer heat and soil organic matter on severity, 387 ; iprodione resistance in Botrytis cinerea from strawberry and blackberry, 396; modeling effects of environmental conditions on HT2 and T2 toxin accumulation in field oat grains, 57; ontogenic resistance to Podosphaera aphanis in strawberry, 954, cover photo: September; Peronospora variabilis in quinoa seed, molecular detection and phylogeny, 379; Phytophthora capsici, competition between pyrimorph-sensitive and pyrimorph-resistant isolates, 269; Phytophthora community structure in Oregon nurseries, 1052;

Plasmopara viticola, geographic distribution of cyrptic species, eastern North America, 692; spatial distribution of SNPs related to fungicide resistance, 604; tripartite interactions in salt marsh dieback, 1070; Verticillium nonalfalfae, pathogenicity and virulence of isolates from Ailanthus altissima, 282, cover photo: March

Ecometagenetics: using to discriminate plantparasitic nematodes from soil communities, 749 Edirisinghe, V. P., S3.36

Edwards, J., S3.47

Edwards, S. G., 57

Effector proteins: Meloidogyne avirulence protein family, multiple ligand-like motifs in, 879

Eggenberger, S. K., 365, S3.37

Eikemo, H., S3.86

Eisenback, J., S3.93

Elad, Y., 150

Elbaz, M., 484

Elci, E., 108

El-Ganainy, S. M., S3.37

El Hadrami, A., S3.9

El-Hawary, M., S3.106

El Jarroudi, M., S3.15, S3.37

Elkahky, M. T., S3.113

El-Kassaby, Y. A., S3.65

Elling, A. A., 497, 1098, S3.37

Elliott, S., S3.181

Ellis, M. A., 692

Ellis, M. L., 1322, S3.37

Elmer, W. H., 1070

El-Shafey, R., S3.55

El-Shetehy, M. H., S3.37

Emery, R., S3.83

Eng, J., S3.141

English, J., S3.96

E-probe diagnostic nucleic acid assay: screening metagenomic data for viruses with, 1125

Eriksmoen, E., S3.167

Ernest, E. G., S3.162

Errampalli, D., S3.38

Erye, M., S3.166

Esca disease: arthropod vectors, 1063

Escriu, F., 1241

Eshel, D., 240

Eskalen, A., S3.182

Eskandari, F. M., S3.160, S3.161

Esker, P., S3.46, S3.58

Esker, P. D., S3.38, S3.111

Espinosa, P., S3.44

Essarioui, A., S3.38, S3.67

Essenberg, M., 1088

Esvelt Klos, K., S3.38

Ethephon: effect on development of gray leaf spot in perennial ryegrass, 614

Etiology: Potato spindle tuber viroid, distribution in petunia floral organs, 964

Evanega, S. D., S3.157

Evans, J. G., S3.38

Evans, K. J., S3.183

Evans, T. A., S3.162, S3.163 
Everhart, S. E., S3.153

Eversole, K., S3.144

Everts, K. L., S3.38, S3.80, S3.162

Evett, S., S3.109

Ewing, L., S3.55

Fabi, A., 387

Fadamiro, H., S3.84

Faedda, R., 851

Fakhoury, A., S3.10, S3.53, S3.84, S3.127,

S3.139

Falardeau, J., 1036

Falconer, L., S3.128

Falconi, C. E., S3.39

Falk, B. W., 108

Fall, L. A., S3.143

Farah, S. C., S3.8

Faris, J. D., S3.58, S3.60, S3.71, S3.154, S3.165

Farrar, J., S3.181

Faske, T., S2.4

Faure, C., 660

Favaro, M. A., 970

Favaron, F., 1201

Feau, N., S3.101

Fei, Z., S3.30, S3.68

Fekak, G., S3.79

Felcher, K., S3.169

Feldman, L., 484

Felice, L., S3.39

Felix, K. C. S., 1175

Feng, C., S3.39

Feng, J., 1078, S3.39, S3.136

Feng, X., 786, 1251, S3.152

Fengycin: effect of membrane lipids on plant sensitivity, 1036

Fenoglio, J., S3.90

Fenstermacher, K. A., S3.148

Ferguson, M., S3.132

Ferguson, M. H., S2.4

Fernandes, F. R., S3.79

Fernández-Ortuño, D., 396, 724

Fernandez-Pavia, S. P., S3.126

Fernando, D., S3.7, S3.12, S3.40, S3.46, S3.74

Fernando, W. G., S3.135

Ferrada, E., S3.33

Ferrada, E. E., S3.40

Ferrandino, F. J., S1.2

Ferrándiz, J. C., 804

Ferrari, M. J., S3.110

Ferreira, C., 416

Ferreira dos Santos, P. C., S3.40

Ferrocino, I., S3.48

Fetz, A., S3.112

Fichtner, E. J., S3.40

Fieland, V. J., 1052, S3.61

Fig mosaic virus: genetic variation and evolution in, 108

Figueroa, M., S3.23, S3.40

Filichkin, S., S3.40

Filion, M., 926

Filippi, M. C. C., S3.47

Firth, A. E., S3.81

Fischer, K. F., 211

Fischer, T., S3.9

Fisher, T., S3.20

Fisher, T. W., S3.40, S3.179

Fitzgibbon, T., S3.166

Flaherty, J. E., S3.52, S3.87

Fleischmann, F., S3.18

Fleites, L. A., S3.41, S3.55

Fletcher, J., S3.82

Flinders Technology Associates, 306

Florence, J., S3.181

Flores, F., S2.4

Flores, F. J., S3.44
Floyd, C., S3.74

Fludioxonil resistance: in Botrytis cinerea isolates

from blackberry and strawberry, 724

Fnu, S., S3.41

Fochs, B., S3.172

Foley, M., S3.54

Folta, K. M., S3.91

Fomitiporia mediterranea: wood rot of citrus, Italy, 851

Fomitopsis spp.: on citrus, characterization of, Italy, 851

Font, M. I., 804

Fontaine, F., 1021

Forbes, G., S3.51, S3.118

Forbes, G. A., 624

Forcelini, B. B., S3.41

Forster, H., S3.3

Fortuna, A. M., S3.138

Fortunato, A. A., 597

Fory, P. A., 436

Foshee, W., S3.84

Foster, C., S3.40

Foster, N., S2.5

Foster, N. M., S2.11

Foster-Hartnett, D., S3.128

Fox, A., S3.41

Fraaije, B. A., S3.41, S3.99

Fragaria $\times$ ananassa: fludioxonil resistance in Botrytis cinerea isolates, 724; genetic variation and gains in resistance to Colletotrichum gloeosporioides, 67; iprodione resistance in Botrytis cinerea, 396; ontogenic resistance to

Podosphaera aphanis, 954; powdery mildew on, cover photo: September

França, S. K. S., 1183

France, R. A., S3.42, S3.47

Franceschi, J., S3.42

Francis, I. M., S2.4

Francisco, C. S., 120

Franco, E. M., S3.11

Francois, M. G., S3.171

Franke, J., 1306, S3.42

Fraser, M. C., S3.42

Frazier, R., S2.5

Frazier, T., S3.161

Frederick, Z., S3.29

Frederick, Z. A., S3.42, S3.122

Freed, G., S3.181

Freeman, J. H., S3.64

Friebe, B., S3.60

Friel, M., S3.111

Friesen, T., S3.36

Friesen, T. L., S3.60, S3.154

Frigulti, T. L., S3.27

Friskop, A., S3.52

Frogeye leaf spot. See Cercospora sojina

Frost, K., S3.42, S3.159

Frost, K. E., S3.172

Frosty pod rot. See Moniliophthora roreri

Fry, W., S1.2

Fry, W. E., S1.4, S3.30, S3.90, S3.149

FTA technology: for DNA-based analyses of

plant-parasitic nematodes, 306

Fu, Y.-B., S3.18

Fuchs, M., 211, 1232, S3.63, S3.77

Fuchs, S., S3.131

Fujie, M., S3.4

Fujiyoshi, P. T., S3.43

Fukuda, S., S3.162

Fulbright, D. W., S3.78

Fulladolsa, A. C., S3.43, S3.169

Fulmer, A. M., S3.155

Fultz, J. E., S2.4

Fumonisin: contamination in Kenyan maize mills, 1221
Fungal effectors: in a multilocus analysis of Fusarium oxysporum from sugar beet, 886

Fungicide mixtures: in the management of fungicide resistance, 1264

Fungicide resistance: with high-risk fungicides in fungicide mixtures, 324; management with fungicide mixtures, 1264; spatial distribution of SNPs related to, 604

Funnell-Harris, D. L., S3.43, S3.169

Fusarium-damaged kernels, 472

Fusarium head blight (FHB): Fusarium-damaged kernels and deoxynivalenol in winter wheat, 472; modeling effects of environmental conditions on HT2 and T2 toxin accumulation in field oat grains, 57; predicting epidemics with boosted regression trees, 702. See also Fusarium graminearum

Fusarium spp.: infection route into Bromus tectorum seeds, 1306; modeling effects of environmental conditions on HT2 and T2 toxin accumulation in field oat grains, 57

-F. acuminatum: crown and root rot of asparagus, soil organic amendments and, 403

- F. graminearum: fitness attributes of isolates with 3-ADON or 15-ADON trichothecene genotypes, 513; Fusarium-damaged kernels and deoxynivalenol in winter wheat, 472;

Pseudomonas chlororaphis subsp. aurantiaca strain Pcho10 as a potential biocontrol agent, 1289; toxigenic capacity and trichothecene production related to aggressiveness in wheat, 357; virulence factors and disease severity on soybean, 1201. See also Fusarium head blight

-F. langsethiae: in oat, 57

-F. oxysporum: crown and root rot of asparagus, soil organic amendments and, 403; from sugar beet, multilocus analysis, 886

-F. oxysporum f. sp. betae: from sugar beet, multilocus analysis, 886

-F. oxysporum f. sp. cubense: resistance in banana, potentiated by silicon, 597

-F. oxysporum f. sp. cucumerinum: control with soil fumigation and Clonostachys rosea, 1314

-F. oxysporum species complex: characterization of isolates from soybean roots, 1329

-F. palustre: tripartite interactions in salt marsh dieback, 1070

-F. proliferatum: crown and root rot of asparagus, soil organic amendments and, 403

-F. redolens: crown and root rot of asparagus, soil organic amendments and, 403

-F. solani: crown and root rot of asparagus, soil organic amendments and, 403

Fusarium yellows: from sugar beet, multilocus analysis, 886

Fuzzy Peronospora Sparsa model, 50

Gabriel, D. W., S3.41, S3.55

Gadea, J., 970

Gadoury, D. M., 692, 954, S3.149

Gaeumannomyces graminis var. tritici: biological control by Pseudomonas fluorescens HC1-07, 248

Gager, J., S1.3, S3.65

Galarneau, E. R., S3.43

Galeana-Sanchez, E., S3.93

Galindo-Sánchez, C. E., S3.183

Gamba, F., 715

Gamliel, A., S3.82

Ganci, M., S3.44, S3.147

Gang, D. R., S3.60

Gang, G., S3.44

Gangneux, C., 1138

Gao, J.-X., 332

Gao, L., 445, S3.48 
Gao, M., S3.46

Gao, Q., 1289

Gao, S., S3.46, S3.68

Gao, Y., S3.154

Garbe, J., S3.19

Garber, N. P., 462

Garcés, G., S3.44

Garcia Aroca, T. G., S2.2

Garcia Suarez, J. A., S3.34

Gardiner, M. M., S3.12

Garfinkel, A., S3.180

Garibaldi, A., S3.48

Gariglio, N. F., 970

Garita, L., S3.82

Garnica, V. C., S3.20, S3.82, S3.83

Garrett, C., S3.71

Garrett, K., S3.51, S3.93, S3.118

Garrett, K. A., S3.44, S3.51

Garrido, P. A., S2.4, S3.44

Garvin, D. F., S3.23, S3.40

Garzon, C., S2.8, S3.82

Garzon, C. D., S2.4, S2.8, S3.44

Gaspar, J. M., 749

Gastelo, M., 624

Gauslaa, E., S3.86

Gautam, P., S3.45

Gazzetti, K., 1201

Gealy, D. R., S3.71

Geary, B., 1306, S3.42, S3.52

Gebremariam, T., S2.11, S3.124

Gehesquiere, B., S3.74, S3.146

Geiser, D. M., S3.148

Geisler, M., S3.84, S3.139

Genetics and resistance: fludioxonil resistance in

Botrytis cinerea isolates from blackberry and

strawberry, 724; Fusarium-damaged kernels and deoxynivalenol in Fusarium-infected winter wheat, 472; genetic analysis of leaf rust resistance in durum wheat, 1322; genetic variation and gains in resistance of strawberry to Colletotrichum gloeosporioides, 67;

histochemical characterization of early response to Cochliobolus sativus in barley, 715 ; identification of a hidden resistance gene in tetraploid wheat, 634; induction of salicylic acid-mediated defense response in perennial ryegrass against Magnaporthe oryzae, 614; late blight resistance in potato genotypes adapted to tropical highlands, 624; leaf rust resistance in wheat breeding lines, molecular mapping, 865; mapping of Phytophthora fruit rot resistance in pepper, 479; pyramiding $B$ genes in cotton and bacterial blight resistance, 1088; role of leaf features in canker disease resistance in Satusma mandarin 'Okitsu', 970; role of primary and secondary infection in host response to Plasmodiophora brassicae, 1078; sexual reproduction in Aspergillus flavus sclerotia in corn, 75; trehalose induction of wheat defenses to powdery mildew, 293; white pine blister rust resistance in limber pine, 163; $\operatorname{Yr} 36$ gene confers partial resistance in wheat to Puccinia striiformis, 871

Genger, R. K., S3.45, S3.169

Gent, D., S3.130

Gent, D. H., S3.45, S3.115 S3.149

Gentit, P., 660

George, S., S3.11

Gerberich, K. M., S3.47, S3.56

Gerik, J., S3.30, S3.69

Gérin-Ouellet, M., 926

Gevens, A., S3.27, S3.42, S3.103, S3.168,

S3.169, S3.170

Gevens, A. J., S3.172

Ghavami, F., S3.60
Ghimire, S., S3.45

Giampetruzzi, A., S3.133

Giblin, C. P., S3.51

Giblin-Davis, R. M., S3.10

Gibson, W. G., S3.100

Gidoin, C., 275

Gieco, J., 1340

Giesbrecht, M., S2.2, S3.45

Giesler, L. J., S3.91, S3.167, S3.174

Gigot, C., S3.45, S3.46

Gil, J., S3.75

Gilardi, G., S3.48

Gilbert, M. T., S3.104

Gilbertson, R., S3.145

Gilbertson, R. L., S3.156

Gilkes, J. M., S3.6

Ginzel, M. D., S3.77

Girard, I. J., S3.12, S3.46

Girotti, J. R., 357

Gitaitis, R., 232, S2.3, S2.9, S3.36

Gitaitis, R. D., 812

Glaubitz, J., S3.153

Glazebrook, J., S3.72, S3.73

Gleason, M., S3.12

Gleason, M. L., S3.12, S3.71

Gleeson, G., S3.46

Glenn, A. E., S2.7, S3.46, S3.114

Glenn, T., S3.153

Globodera spp.: effector proteins, 879

Glockling, S. L., S3.113

Gloer, J., S3.133

Glover, K., S3.2, S3.165

Glover, K. D., S3.58

Glycine max: Bean pod mottle virus epidemics, geospatial and temporal analysis, 365;

characterization and quantification of

Phakopsora pachyrhizi in, 86; characterization

of Fusarium oxysporum species complex isolates from plant roots, 1329; effect of silicon on resistance to Cercospora sojina, 1183; Fusarium graminearum virulence factors and disease severity, 1201; inhibitory effects of stilbenes on in vitro growth of soybean pathogens, 843; TaqMan-qPCR assay for Cercospora kikuchii in leaves, 1118

Glynn, N., S3.100

Gmitter, F. G. Jr., 970

Godoy-Lutz, G., S3.90

Goeke, S., S2.11

Goeke, S. C., S2.10

Gold, S. E., S3.46

Goldberg, J., S3.31

Golden, B., S3.73

Golden, B. R., S3.129

Gomez-Montano, L., S3.44

Gonçalves, M. G. M., 1183

Gonçalves-Zuliani, A. M. O., S3.18, S3.86

Gongora Castillo, E., S3.130

Gonsalves, D., S3.162

Gonzalez, C. F., S3.140

Gonzalez, E. T., S3.168

Gonzalez, J., S3.165

Gonzalez, L., S3.97

Gonzalez, M., S3.63

Gonzalez, Y., S3.33

Gonzalez-Hernandez, J. L., S3.58

González Venegas, J. P., S3.46

Goodwill, T. R., S3.50

Goodwin, P. H., S3.38

Goodwin, S. B., S3.154

Gordon, S., S3.40

Gordon, T. R., S3.71, S3.97, S3.116

Gorham, S., S3.87

Goss, E. M., S2.10, S3.9, S3.62, S3.126

Gossen, B., S3.117
Gossen, B. D., 1078, S3.29, S3.31, S3.32, S3.53, S3.77

Gossypium hirsutum: effects of Meloidogyne

incognita and Thielaviopsis basicola on growth and root morphology, 507; pyramiding $B$ genes and bacterial blight resistance, 1088

Gottwald, T. R., 575, S3.16, S3.73, S3.98

Goyer, C., 926

Grabau, Z., S3.25

Grabke, A., 396, 724

Grabow, B. S., S3.47

Graf Grachet, N., S3.47

Graham, J. H., S3.16, S3.47, S3.56, S3.131

Grant, J. F., S3.30, S3.48

Grapevine red blotch-associated virus: analysis in the U.S., 1232

Grapevine red blotch disease: Grapevine red blotch-associated virus and, 1232

Grapevine trunk diseases: arthropod vectors, 1063, cover photo: October

Grapevine vein clearing virus: genetic diversity and tissue and host specificity, 539

Grapevine virus A: genetic diversity, Washington and California, 548

Graphocephala atropunctata: transmission of Xylella fastidiosa, 27

Graves, A. D., S3.135

Gray, J., S2.8, S3.158

Gray, S. M., S3.70, S3.141, S3.180

Green, K., S3.70

Green, S., S3.88

Greenland, A., 871

Greer, A. M., S3.113

Gregorio-Cipriano, R., S3.126

Grenier, C., S3.93

Grey, T., S2.2

Griffin, G. J., S3.148

Griffiths, S., S3.154

Grim, C. J., S3.55

Grinbergs, D. E., S3.42, S3.47

Grisham, M., S3.128

Grisham, M. P., S3.47

Groom, T., S3.13

Großkinsky, D. K., 1283

Groth-Helms, D., S3.73

Grove, G., S3.45

Groves, R., S3.159, S3.169

Groves, R. L., S3.45, S3.112, S3.167, S3.178

Grunwald, N. J., 1052, 1107, S3.57, S3.61,

S3.126, S3.142, S3.149, S3.153

Grusak, M., S3.26

Gu, G., S3.98

Guan, L., 158

Gubler, W. D., S3.116, S3.182

Gudmestad, N. C., 40, S3.106

Guerrero, O., S3.184

Guest, D., 933

Gugino, B. K., S1.2, S1.4, S1.5, S3.92, S3.142, S3.161

Guilhabert-Goya, M., S3.155

Guimarães, R. A., S3.47

Gulari, E., S3.159

Gulden, R., S3.49

Gullino, M. L., S3.48

Gulya, T., S3.52

Gulya, T. J., S3.98

Gundersen, B., S3.181

Guo, B., S3.132

Guo, H., S3.146

Guo, J.-H., 248

Guo, L., S3.31, S3.48

Guo, L. Y., S3.125

Guo, Q., 539

Gurung, S., 641, 779

Gutierrez-Gonzalez, J., S3.23 
Guyot, J., 586

Guzman, M. L., S3.21

Gwinn, K. D., S3.20

Hadi, B., S3.116

Hadziabdic, D., S3.30, S3.48

Hagan, A., S3.17

Hagan, A. K., S3.49, S3.68

Hagberg, I., 1036

Hageman, B., S3.35

Hagstrum, D., S3.51

Haile Mehari, Z., 150

Hajeri, S., S3.133

Hajihassani, A., S3.49

Hajimorad, M. R., S3.49

Hajimorad, R., S3.151

Halbrendt, N., S1.5

Halfman, B., S3.111

Hall, D. G., S3.51

Halleen, F., 1063

Halloran, J. M., S3.66

Halterman, D., S3.79, S3.168, S3.172

Halterman, D. A., S3.43, S3.59

Halvorson, J., S3.49, S3.170

Ham, J. H., S3.50, S3.87, S3.108

Hamada, M. S., 1289

Hambleton, S., S3.50, S3.59

Hamelin, R. C., S3.65, S3.101, S3.153

Hamilton, J., 436

Hamm, P. B., S3.181

Hammerschmidt, R., S3.58

Hammett, C., S3.50, S3.106

Hammond, J., S3.145

Hammond, R. W., S3.146

Hammond, T. M., S3.170

Hamon, H. G., S2.2

Han, A., S3.162

Han, B., S3.58

Han, C. S., S3.125

Han, C. U., S3.58

Han, E. J., S3.60, S3.107

Han, G. D., 834

Han, M., S3.125

Han, X., S3.126

Handoo, Z. A., S3.22

Hanes, D., S3.55

Hannan, A., S3.50

Hansen, E., S3.153

Hanson, B., S3.69

Hanson, B. D., S3.30

Hanson, L. E., S3.50, S3.55, S3.176

Hanson, M., 886, S3.128

Hao, J., S1.3, S1.4, S3.55, S3.136, S3.137, S3.176

Haq, I. U., S3.9

Harbach, C. J., S3.170

Harding, M., S3.57

Hardy, C. M., 749

Hardy, T. N., S2.9

Harmon, C. L., S2.7

Harmon, P. F., S2.7

Harper, A., S3.89

Hartel, C., S3.135

Hartley, S., S3.85

Hartman, D., S3.184

Hartman, G. L., 86, 843, 977, S3.24, S3.90, S3.170

Hartung, J. S., S3.101

Harvey, A. M., S3.170

Harvey, J. W., 1221

Hashem, F., S3.67

Hashsham, S., S3.159

Hassell, R., S3.117

Hatsugai, N., S3.73

Haudenshield, J., S3.100

Haugen, L., S3.104
Hausbeck, M. K., 479, S3.84, S3.176

Havis, N. D., S3.91

Hawes, M., S3.119, S3.177

Hawkins, A. E., S3.50

Hawkins, L., S3.140

Hawkins, N. J., S3.41

Hay, F. S., S3.90, S3.91, S3.105

Haygood, R. A., S2.5

Hayslett, M., S3.59

Hebert, J., S3.74, S3.146

Hegeman, A. D., S3.82

Hein, G., S3.131, S3.152

Heiniger, R., S3.68

Held, B. W., S3.51

Hempfling, J. W., S3.13, S3.160

Henn, A., S3.2

Henne, D., S3.64, S3.68

Herbert, D. A., S3.79

Hernandez, V., S3.184

Hernandez-Martinez, R., S3.179, S3.183

Hernandez Nopsa, J., S3.51, S3.118

Herrero, M., S2.4, S3.44

Heterodera spp.: effector proteins, 879

$-H$. avenae: discrimination from soil

communities using ecometagenetics, 749

-H. schachtii: FTA technology for DNA-based

analyses, 306; nondestructive imaging in planta, 497

Heuberger, A. L., S3.99, S3.171

Heungens, K., S3.74, S3.146

Hewezi, T., 879

Hiebert, C., S3.19

Highland, B., S3.51

Highland, H. B., S3.54

High-risk fungicides: in fungicide mixtures and

the problem of fungicide resistance, 324

High-throughput automated image analysis: to measure quantitative virulence of Zymoseptoria tritici, 985

Hijaz, F. M., 15

Hilf, M. E., 15, S3.51

Hill, C. B., 86, 843

Hill, J., S3.101

Hill, T. A., 479

Hillis, V., S3.12

Hirsch, P. R., S3.99

Hirsch, R. L., S3.52, S3.87

Hldaky, L., S3.128

Ho, T., S3.32, S3.52, S3.117

Hobbelen, P., 1264

Hobbs, H. A., S3.133

Hockemeyer, K. R., S3.52

Hodda, M., 749

Hoenisch, R. W., S3.77

Hoerth, S. E., S3.173

Hoffmann, F., S2.11, S3.122

Hoffmann, V., 1221

Hogan, C., S3.75

Hogenhout, S. A., S3.150

Holbrook, C. C., S2.7

Holdsworth, W., S3.26

Hole, H., S3.86

Holladay, W. T., S3.183

Holland, R., S2.2, S3.28, S3.134

Hollandbeck, G. F., S3.170

Hollier, C., S2.6

Hollier, C. A., S2.5, S3.149

Hollingshead, A., S3.52

Holloway, P. S., S3.180

Holmberg, A., S3.112

Holmes, E. C., S3.110

Honesty, S., 539

Hong, C., S3.133, S3.147

Hong, C. Y., S3.69

Hong, J. C., S3.52
Hong, S. J., S3.60, S3.107

Hooftman, M., S3.78

Hooks, C. R., S3.80

Hordeum vulgare: histochemical characterization of early response to Cochliobolus sativus

infection, 715

Horn, B. W., 75

Horsfall-Barratt (H-B) scale, 575

Hotchkiss, M. W., S3.15, S3.16

Hovmøller, M. s., 1042

Hoyt, P. R., 1125

Hsu, C. Y., S3.73

HT2 toxin: accumulation in field oats, 57

$\mathrm{Hu}$, B., 1130

$\mathrm{Hu}$, C. H., S3.71

Hu, J., 269, S3.32, S3.79, S3.112, S3.162

Hu, M., S3.79

$\mathrm{Hu}, \mathrm{W} ., 1289$

Huang, H., S3.115

Huang, T. P., S3.25

Huanglongbing. See 'Candidatus Liberibacter asiaticus'

Hubbard, A., 871

Huber, D. J., S3.113

Huber, L., S3.46

Huerta, A. I., S3.156

Huerta Arrendondo, I. A., S1.2

Huffaker, A., S3.139

Huffman, V., S3.105

Hughes, M. A., S3.148

Hulcr, J., S3.147, S3.148

Hulvey, J., S1.2, S1.3, S1.6

Humann, J. L., S3.77

Humann, R., S3.52

Hung, R., S3.67

Hunger, R. M., S3.47

Hupalo, D., S3.161

Hurd, K., S2.4

Huseth, A., S3.159

Hussey, R. S., 879

Huzar Novakowiski, J., S3.166

Hwang, S. F., S3.22, S3.39, S3.53, S3.77, S3.136, S3.138

Hwang, S.-F., 1078

Hwang, S. F. F., S3.42

Hyma, K., S3.152

Hyon, G.-S., 634

Hypersensitive reaction: in barley response to Cochliobolus sativus infection, 715

IAN (3-indolylacetonitrile), 134

Ibarra, M. A., S3.94

Ibarra Caballero, J. R., S3.53, S3.135

Iberkleid, I., 484

Idris, A., S3.53

Idris, A. M., S3.6

Iftikhar, R., S3.107

I gene-bearing common bean: temperatureinsensitive necrosis caused by a recombinant Bean common mosaic virus, 1251

Iida, Y., S3.154

Ilic, S., S3.142

Inch, S., S3.148

Inderbitzin, P., 564, S3.53

3-Indolylacetonitrile (IAN), 134

Induced systemic resistance: induction of priming-mediated ISR in cucumber against Colletotrichum orbiculare, 834

Inglis, D. A., S3.181

Ingram, J. T., S3.70

Inguagiato, J., S3.143

Innes, L., S1.1

Inoue, Y., 634

Inoue-Nagata, A. K., S3.79

Ionescu, M., 27 
Ippolito, A., 851

Iprodione resistance: in Botrytis cinerea from strawberry and blackberry, 396

Irby, J. T., S3.129

Ireland, D., S3.86

Irey, M., S3.100

Iriarte, F., S3.9

Isakeit, T. S., S2.6

Ishii, H., S3.53

Ishimaru, C. A., S3.72, S3.73, S3.74, S3.78,

S3.113, S3.173

Islam, K. T., S3.53

Islam, M. R., S3.138

Islam, M. S., S3.54

Islam, S. Z., S3.10

Ismaiel, A., S1.5, S3.163

Ismaiel, E., S3.74

Issac, Y., S3.82

Ivors, K., S3.17, S3.44, S3.77, S3.147

Iyer-Pascuzzi, A. S., S3.177

Jacaranda mimosifolia: leaf extracts, efficacy against wheat leaf rust, 933

Jackson, R. W., S3.99

Jackson-Ziems, T. A., S3.66

Jacobi, W., S3.35

Jacobs, J., S3.127

Jacobs, J. J., S3.54

Jacobs, J. M., S3.72

Jacobsen, B. J., S3.54

Jacoby, T. P., S2.5

Jacquot, E., S3.98

Jagdale, G. B., S3.183

Jahn, C. E., S3.171

Jahn, M. M., S3.166

Jaime, R., S3.54

Jaime-Garcia, R., S3.139

Jain, M., S3.41, S3.55

Jain, S., S3.19, S3.54

Jan, F. J., S3.115, S3.136

Jansky, S., S3.5, S3.169, S3.172

Jansky, S. H., S3.43, S3.45

Jara, S., S3.33

Jaraba, J., 507

Jardine, D. J., S3.171

Jarosz, A. M., S3.62, S3.78

Jarroudi, M. E., S3.26

Jarvis, K. G., S3.55

Jasmonic acid (JA): effect on development of gray

leaf spot in perennial ryegrass, 614

Javan-Nikkhah, M., S3.4

Jayaraman, D., S3.122

Jecmen, A., S2.5, S2.9

Jee, H. J., S3.60, S3.107

Jelkmann, W., 453

Jensen, B., S3.111

Jeon, Y. H., S3.26, S3.27, S3.60

Jeun, Y. C., 834

Jeyaprakash, A., S3.114

Ji, G.-H., 672

Ji, P., S3.55, S3.92

Ji, Z.-Y., 672

Jia, Y., 762, S3.55, S3.70, S3.71, S3.124, S3.125

Jiang, H., S1.3, S3.55

Jiang, J., S3.166

Jiang, Q., S3.55

Jiang, T., S3.132

Jiang, Z., S3.131

Jimenez, D. R. C., 1322

Jimenez, P., S3.97, S3.99

Jimenez, V. M., S3.149

Jimenez-Diaz, R. M., 650, S3.153

Jimenez-Gasco, M., S3.12, S3.153

Jimenez-Gasco, M. M., 379, 650

Jin, F., 472
Jin, Y., S3.56

Jing, L., S3.85, S3.173

Jo, Y. K., S2.5, S3.82

Jochum, C., S3.114, S3.134

Johnson, C., S3.93

Johnson, D., S3.96

Johnson, D. A., S3.42, S3.158, S3.185

Johnson, E. G., 15, S3.47, S3.56, S3.131

Johnson, G. C., S3.162, S3.163

Johnson, J. L., S3.56

Johnson, K., S3.52

Johnson, K. B., S3.56

Johnson, R. M., S3.47

Johnson, S. B., S1.3, S1.4

Johnson, W. G., S3.170

Johnston, C. M., S3.95

Johnston, M. T., S1.2, S3.158

Joly, D. L., S1.3

Jones, J., S3.178

Jones, J. B., S2.10, S3.64, S3.77, S3.91, S3.114, S3.119

Jones, M. W., 1360

Jones, R., S3.29, S3.56

Jones, S. J., S3.91

Jones, S. S., S3.180

Jordahl, J., S3.52

Jordan, J., S2.3

Jordan, K., S3.121

Jordan, R., S3.81

Jordan, S., S3.168, S3.169

Jordan, W. J., S2.5, S3.28, S3.56, S3.59

Jordon, A., S3.128

Joseph, L., S1.2, S3.149

Joshua, J. O., S3.56

Ju, H., S3.57

Juglans regia: colonization of dormant buds by

Xanthomonas arboricola pv. juglandis, 1163

Julian, P., S3.149

Jumpponen, A., S3.44, S3.93

Jun, Z. Y., S3.57

Jung, G., S1.2, S1.3, S1.5, S1.6, S3.5

Junior, M. L., S3.109

Juzwik, J., S3.77, S3.96, S3.104, S3.132, S3.148

Kabbage, M., S3.19, S3.95, S3.130, S3.171

Kachapulula, P. W., S3.57

Kachroo, A., S3.37, S3.69, S3.112

Kachroo, P., S3.37, S3.69, S3.112

Kago, L., S3.45

Kakongi, N., 240

Kalischuk, M., S3.57

Kalogeropoulou, P., 347

Kaminski, J. E., S3.159

Kampitova, G., S3.133

Kamvar, Z. N., S3.57

Kanaan, M., S3.100

Kanaskie, A., S3.153

Kandel, P. P., S3.58

Kandel, Y. R., S3.58

Kang, H. J., S3.58

Kang, S., S3.1, S3.61

Kaniszewski, L. J., S3.58

Kaplan, J., S3.12

Karakaya, A., S3.23

Karaoglanidis, G. S., 347, S3.62, S3.63

Karasev, A. V., 786, 1251, S3.70, S3.152, S3.180, S3.182, S3.183

Kariyawasam, G., S3.58

Karki, H. S., S3.108

Kartanos, V., S3.14, S3.59

Kassatenko, I., S3.50, S3.106

Kasson, M. T., 282, S3.148

Kasun, G., S3.40

Katagiri, F., S3.73

Kaur, K., S2.6
Kawaguchi, A., S3.59

Kawamura, R., 1001

Kawasaki, T., S3.4

Kawate, M., S3.79

Kawchuk, L., S3.57

Kefialew, Y. W., S3.11

Kegel, F. R., S3.129

Kegley, A., 163

Keller, K. E., S3.33, S3.117

Keller, N., S3.176

Kelly, H., S3.59

Kelly, H. M., S3.28, S3.56

Kelsey, C., S2.11, S3.124

Kema, G. H. J., 95

Kemerait, R., S3.68, S3.132

Kemerait, R. C., S2.7, S3.155

Kendra, P. E., S3.21

Kennelly, M., S3.44, S3.174

Kenworthy, K. E., S3.10

Keremane, M. L., 257, S3.121

Kern, A., S3.161

Kerschbamer, C., S3.105

Kersey, C. M., S3.35

Kertho, A. O., S3.171

Kesanakurti, P., S3.59

Khabbaz, S. E., S3.1

Khan, I. A., 257, S3.9

Khan, M., S3.9

Khan, M. F., S3.17, S3.70, S3.157

Khan, S., S3.110

Khanal, C, S2.6

Khanal, C., S3.59

Khankhum, S., S3.59

Khatabi, B., S3.49

Khatri, K., S2.6

Kianian, P., S3.60

Kianian, S., S3.60

Kielsmeier-Cook, J., S3.60

Kikkert, J. R., S1.1

Kilcrease, J., S3.95, S3.184

Killiny, N., 15, 27

Kim, E. N., 834

Kim, G., S3.161

Kim, H.-J., S3.108

Kim, H.-W., S3.108

Kim, J., S3.44, S3.64

Kim, J.-S., 993

Kim, K. D., 834

Kim, K.-H., 993

Kim, K. S., 50

Kim, M. J., S2.4, S3.60, S3.107

Kim, M.-K., 993

Kim, S. H., S3.61

Kim, T., S3.58

Kim, T. S., S3.64

Kim, W., S3.60

Kim, Y., S3.58

Kim, Y. K., S3.60, S3.107

Kim, Y. M., S3.60, S3.154

Kim, Y. S., S3.58, S3.60

Kinard, G., S3.81, S3.93

Kingdom Gibbard, H., S3.150

Kingsley-Richards, S. L., S1.5

Kinkel, L., S3.8, S3.38, S3.88

Kinkel, L. K., S3.6

Kinkel, L. L., S3.39, S3.67

Kirk, W. W., S3.55, S3.100, S3.113, S3.168,

S3.175, S3.176

Kirkpatrick, B., S3.92, S3.127

Kirkpatrick, T. L., 507, S3.113

Kirkwood, J. S., S3.171

Kisha, T., S3.35

Kistler, H. C., S3.20, S3.38, S3.48, S3.67, S3.134

Kitajima, E. W., 804

Klamer, D., S3.94 
Klamer, J., S3.94

Klapste, J., S3.65

Klein, A., S3.81, S3.173

Klemsdal, S. S., S2.4

Klingeman, W. E., S3.77

Klink, H., S3.61, S3.122

Klittich, C. J. R., S3.61

Kloepper, J. W., S3.71, S3.132

Klosterman, S. J., 1349, S3.61, S3.180

Kluepfel, D., S3.114

Knaus, B. J., 1052, S3.61, S3.149, S3.153

Kness, A. A., S3.162

Knoblauch, M., 497

Knudsen, G. R., S3.30

Kobayashi, K., S3.85

Kodati, S., S3.61

Koebnik, R., 520

Koehler, A., S3.62

Koenen, B. S., S3.78

Koenning, S. R., S3.38

Koga, L., S3.170

Koike, S., S3.61, S3.75

Koike, S. T., 1349, S3.180

Koishibayev, M., S3.36

Koita, O., 520

Kojima, H., 313

Kokalis-Burelle, N., S3.52

Kokhmetova, A., S3.133

Kolawole, O. O., S3.62

Kolb, F. L., S3.15

Kolmer, J., S3.41, S3.62

Kolmer, J. A., 865

Kolp, M., S3.62

Komm, D. A., S3.62

Konstantinou, S., S3.62, S3.63

Koo, C., S3.162

Korth, K., S2.8

Korth, K. L., S3.150

Kosta, K., S3.105

Kotcon, J., S3.163

Kouadio, L. A., S3.15, S3.37

Kouassi, N., S3.63

Kovalski, I., S3.8, S3.91

Koym, J., S3.68

Krause, M. S., S3.144

Krebs, S., S3.63

Krenz, B., 1232, S3.63

Kressin, J., S3.109

Kreuze, J., S3.51

Kriss, A. B., S3.16

Kromann, P., S3.118

Kronlein, M., S3.159

Kroschel, J., S3.51

Krueger, W. H., S3.40

Kruger, G. R., S3.66

Kthiri, D., 1322

Kuldau, G. A., 614

Kumar, S., S3.110

Kundu, S., S3.184

Kunoh, H., S3.108, S3.119

Kunta, M., S3.5, S3.63, S3.64, S3.121

Kunta, M. B., S3.102

Kuntz, D. R., S3.170

Kunwar, S., S3.64

Kurdyla, T., S2.6

Kurjan, J., S3.84

Kurle, J., S3.19, S3.94

Kurle, J. E., S3.64, S3.106, S3.175

Kutcher, H. R., S3.36

Kutcher, R., S3.18

Kutluk Yilmaz, N. D., S3.17

Kuwitzky, B., 886, S3.128

Kuzmanovic, N., S3.64

Kwabena Osei, M. K., S3.156

Kwack, M. S., 834
Kwak, H.-R., 993

Kwak, Y., S3.44

Kwak, Y. S., S3.64

Kwon, J. H., S3.64

Labbe, N., S3.20

LaBorde, C., S3.100

Lactuca sativa: clonal expansion of Verticillium dahliae in, 641; Verticillium wilt, model for multiseasonal spread of, 908

Laflamme, G., S1.1

Lalancette, N., S1.3, S3.65

La Mantia, J., S3.65

LaMarsh, K. A., S1.4

Lamb, M. C., 75

Lambdin, P. L., S3.30, S3.48

Lamichhane, J. R., 387

Lamine Fall, M., S3.65

LaMondia, J. A., S3.147

Lamour, K., S3.28, S3.59

Lamppa, R., S3.49, S3.170

Lan, P., S3.65, S3.93

Land, C. J., S3.65

Lane, S., S3.87

Laney, A. G., S3.150

Lang, P., S3.25

Lange, D., S3.117

Lange, H. W., S1.1

Langemeier, C. B., S3.66

Langston, D., S2.3, S2.7, S2.8, S3.36

Langston, D. B., 812, S3.103

Langston, D. B., Jr., 232

Lanier, J., S3.103

Lanning, K. K., S3.66

Lanza, F. E., S3.66, S3.112

Lanze, C. E., S3.66

Laplace, D., 586

Larignon, P., 1021

Larkin, R. P., S3.66

Larrainzar, E., S3.122

Larsen, M., S3.126

Lasiodiplodia theobromae: carbohydrate metabolism changes in Prunus persica infected with, 445

Late blight resistance: in potato genotypes adapted to tropical highlands, 624

Latin, R. X., S3.52

Latorre, B. A., S3.33, S3.40, S3.92

Laughlin, D. A., S2.6

Launay, J., 586

Laval, K., 1138

Lawrence, A., S3.2, S3.101

Lawrence, D. P., S3.43, S3.67

Lawrence, K., S3.65, S3.66, S3.112

Lawrence, K. S., S3.36, S3.108, S3.132

Le, V. H., S3.86

Leach, J., 520, S3.120

Leach, J. E., 436, S3.119, S3.166, S3.171

Leaf gas exchange: effects of silicon on in Pyricularia oryzae infected wheat, 143

Leaf rust. See Puccinia triticina

Leandro, L. F., 1322, S3.29, S3.116

LeBlanc, N., S3.38, S3.67

LeBoldus, J., S3.2

LeBoldus, J. M., S3.36

Lebreton, L., 586

Leconte, M., 1042

Ledbetter, C., S3.152

Lee, C. H., S3.136

Lee, D., S3.132

Lee, H. S., S3.64

Lee, I. H., S3.26

Lee, I. M., S3.161

Lee, J. H., S3.67, S3.113

Lee, L., S3.67
Lee, M., S3.60

Lee, M. H., S3.107

Lee, P. A., S2.7

Lee, R. F., 257, S3.121

Lee, S., S3.67, S3.165

Lee, S. D., S3.64

Lee, S. G., S3.26

Lee, T., S3.140

Lee, Y. J., S3.81, S3.173

Lee, Y. M., S3.27

Lefebvre, A., S3.22

Lefebvre, F., S1.3

Legg, J., S3.51, S3.118

Legge, W. G., S3.120

Lehman, B., S1.5

LeJeune, J. T., S3.142

Lencowski, J., S3.106

Leng, Y., S3.4, S3.67

Leslie, J., S3.51, S3.173

Leslie, J. F., S3.167

LesR protein, 224

D-Leucine, 134

Levesque, A., S2.4, S3.44

Lévesque, C. A., 422, S3.113

Levi, A., S3.117

Levy, J. G., S3.68

Levy, L., S3.32, S3.63, S3.92, S3.130

Levy, M., 240

Lewandowski, M. M., S3.68

Lewis, C., 1052

Lewis, M. H., S3.68

Lewis, P., S3.33

Lewis, R., S3.144

Lewis Ivey, M., S3.73, S3.142

Li, B., S1.4, S3.21

Li, C., 762

Li, F., S3.65

Li, G. H., 445

Li, H. X., S3.68

Li, J., 134, 762, S3.69, S3.79, S3.83

Li, M., S3.131

Li, P. F., S3.11

Li, R., S3.65, S3.68, S3.93

Li, S., 794, S3.69

Li, S.-D., 1314

Li, W., S3.63, S3.69

Li, X., 396, 724, S3.50, S3.85

Li, Y., S3.14

Li, Z., 445, 672

Liang, C., S3.79

Liang, M., 158

Liban, S., S3.40

Lichter, A., 240

Lichtner, F., S3.69

Lichty, J., S3.110

Liesner, L., S3.54

Lilium: antimicrobial trait of LsGRP1 protein, 340; LsGRP1 protein dual subcellular localization, 1012

Lillis, J., S3.152

Lily leaf blight, 1012

Lim, G. H., S3.69

Limber pine: white pine blister rust resistance, 163 , cover photo: February

Lin, C. H., S3.156

Lin, C.-H., 340, 1012

Lin, D., S3.21

Lin, H., S3.140

Lin, M., S3.125

Lin, Y. H., S3.69, S3.70

Lin, Y. T., S3.136

Lind, J., S3.70

Lind, J. M., S3.171

Lindow, S., 1163

Lindow, S. E., 27 
Lindqvist-Kreuze, H., 624

Ling, B., S3.137

Ling, K. S., S3.68

Linsell, K. J., 174

Lipase: Fusarium graminearum disease severity in soybean and, 1201

Lipoxygenase: activation in peanut seed cultivars colonized by Aspergillus parasiticus, 1340

Little, C. R., S3.11, S3.90, S3.167

Little, E., S3.13

Liu, C., S3.126

Liu, F., 224, 1130

Liu, H. I., S3.26

Liu, K., S3.71

Liu, M., S3.50

Liu, P., S3.32, S3.126

Liu, Q., S3.70, S3.71

Liu, S.-C., 575

Liu, T., 332

Liu, W., 897, S3.25

Liu, X., 269, S1.4, S3.21, S3.32, S3.83, S3.126

Liu, Y., 897, S3.70, S3.71, S3.158, S3.164

Liu, Z., S3.58, S3.60, S3.71, S3.134, S3.154, S3.165, S3.175

Llorente, I., 1192

Lloyd, M., S3.71

Lo, C.-C., S3.125

Lobo, M., S3.72

Lockhart, B., S3.81

Lockhart, B. E. L., S3.129

Loconsole, G., S3.133

Loladze, A., 1322

Lolium perenne: induction of salicylic acid-

mediated defense response against Magnaporthe oryzae, 614

Lombard, K., S1.4, S3.83

Lookabaugh, E., S3.72

Lopes, C. A., S3.22

Lopes, J. R. S., 416

Lopes, S. L., S3.109

López, M. M., 804

Lopez, P., S3.115

Lopez, S. M., S3.11

Lopez-Escudero, F. J., S3.120

Lopez-Nicora, H. D., S3.72

Lord, R. J., S3.30

Lori, G. A., 357

Loria, R., S2.4

Lou, W., S3.98

Louters, M., S3.128

Louws, F. J., S3.109, S3.129

Louzada, E. S., S3.63, S3.64, S3.121

Lowe, T. M., S3.72, S3.172

Lozovaya, V. V., 843

LsGRP1 protein: antimicrobial trait, 340;

characterization of dual subcellular localization in Lilium, 1012

Lu, F., S3.153

Lu, J., 692

Lu, L., 762

Lu, S. E., S3.73, S3.74

Lu, Y., S3.72, S3.73

Luangkhot, J., S3.66

Lubell, M., S3.12

Luis, J. A., S2.7

Luis, J. M. S., S2.7

Luis-Arteaga, M., 1241

Lujan, P. A., S3.184

Lum, A. B., S3.87

Lunt, T., S3.73

Luo, H., S3.29

Luo, J., S3.41

Luo, L., S3.79, S3.83

Luo, W., S3.73

Luo, Y., S3.3
Lupien, S. L., S3.35

Lurvey, E. L., S1.3

Luster, D. G., S3.76, S3.104

Lutes, L., S3.73

LuxR proteins: roles in Lysobacter enzymogenes strain OH11, 224

Lygin, A. V., 843

Lynch, S. C., S3.182

Lysobacter enzymogenes strain $\mathrm{OH} 11$ : roles of solo LuxR in, 224

Lytle, M., S3.184

M5HF2C toxin: cloning of the gene required for biosynthesis in Cochliobolus lunatus, 332

Ma, B., S3.101

Ma, J., 507

Ma, L., S3.74

Ma, L. J., S3.31, S3.48

Ma, L.-J., 472

Ma, W., 158, 188

Ma, X., S3.73

Ma, Y., S3.26, S3.134, S3.172

Ma, Z., 1289, S3.126, S3.136

Mabagala, R. B., S3.83

MacDonald, W., S3.163

MacGuidwin, A., S3.73, S3.166, S3.167

Machado, M. A., S3.86

Maciel, J. L. N., 95, S3.23

Mackels, C., S3.15, S3.37

MacLean, A. M., S3.150

Macroarrays: RT-PCR/macroarray multiplex platform to profile viral infections in grapevine, 211

Macrophomina phaseolina: inhibitory effects of stilbenes on in vitro growth, 843

Madani, M., S3.117

Madden, L. V., 57, 702, S3.102

Madenova, A., S3.133

Maes, M., 520

Maffia, L. A., S3.19, S3.20, S3.23, S3.82, S3.83, S3.112

Magnano di san Lio, G., 851

Magnaporthe oryzae: induction of salicylicmediated defense response in perennial ryegrass, 614; population structure and pathotype diversity, Brazil, 95; rice resistance gene $P i$-ta, effectiveness and durability in China, 762

Magnin-Robert, M., 1021

Magnusson, C., S3.116

Maguire, I., S3.115

Mahaffee, W. F., S3.118

Mahoney, J., S3.141

Mahovic, M., S3.87

Maier, T. R., 879

Main, D., S3.77

Maize chlorotic dwarf virus: in maize and

Johnsongrass in southern Ohio, 1360

Maize dwarf mosaic virus: in maize and

Johnsongrass in southern Ohio, 1360

Maki, S. L., S3.87

Malapi-Wight, M., S1.5, S3.74, S3.146, S3.162, S3.163

Malbrán, I., 357

Malcolm, G. M., 650

Malinoski, M. K., S3.27

Mallik, I., 40

Maloney, K., S3.133

Malus $\times$ domestica: antagonistic interactions of

'Candidatus Phytoplasma mali' strains in, 453 Malvick, D., S3.74, S3.94, S3.181

Malvick, D. K., S3.78, S3.106, S3.173, S3.175

Mamiro, D. P., S3.156

Maňasová, M., 306

Mandal, M. K., S3.112
Mandel, M. A., S3.132

Mangifera indica: responses to Ceratocystis

fimbriata infection, 820

Maniruzzaman, M., S3.172

Manolii, V. P., S3.22, S3.138

Manrique, N., S3.169

Mansfield, M. A., S3.142

Mansfield, S. D., S3.65

Mansouripour, S. M., S3.74

Manter, D., S3.5

Mao, Q., 897

Mao, S., S3.74

Maoka, T., 313

MAP kinase: Fusarium graminearum disease severity in soybean and, 1201

Mar, T., 897

Maragos, C., S3.140

Marais, A., 660

Marano, M. R., 970

Marcelletti, S., 1274

Marchand, G., S3.132

Marchenko, O., S3.79

Marcillo, V. F., S3.44

Marek, M., 306

Marek, S., S2.8, S3.82

Marek, S. M., S2.4

Mariano, R. L. R., 1175

Markell, S., S3.52

Markoglou, A. N., 347

Marks, M. E., S3.172

Marois, J., S3.11

Maroon-Lango, C., S3.128

Marra, R. E., S3.146, S3.147

Marroquin-Guzman, M. R., S3.74

Marshall, D. S., S3.75

Martelli, G. P., S3.146

Martin, A. C., S3.82

Martin, D. K., S3.148

Martin, F., S3.1, S3.75

Martin, F. N., 733, 1349, S3.61, S3.75, S3.80, S3.98, S3.100

Martin, K. J., S3.52

Martin, M. D., S3.104

Martin, R., S3.94

Martin, R. R., S3.33, S3.66, S3.117, S3.146

Martin, S. B., S3.107

Martínez, C., 804

Martinez-Espinoza, A. D., S3.75

Martins, S. C. V., 34

Maruthachalam, K., 779

Masai, M., S3.52

Mashaheet, A. M., S3.75

Masler, E. P., S3.172

Massa, A., S3.169

Massenz, M., S3.105

Masuta, C., 1001

Matheron, M. E., S3.75

Matic, S., S3.48

Matsumoto, T., S3.162

Matsushita, Y., 964

Matthiesen, R., S3.76

Mauchline, T. H., S3.99

Mauck, K., S3.151

Mauzey, S. J., S3.76, S3.104

Mavrodi, D. V., 248

Mavrodi, O. V., 248

Mavrodieva, V. A., S3.130

May, D., S3.76, S3.182

May, S. R., S3.76

Mayberry, L., S3.94

Mayberry, L. K., S3.174

Mayfield, D. A., S3.29

Mayorquin, J. S., S3.182

Mayton, H. S., S3.149

Mazourek, M., S3.26 
Mazzola, M., S3.4

Mbega, E. R., S3.156

Mbofung, G. Y., S3.2

Mc16D10L effector gene: RNA interference of confers resistance against Meloidogyne chitwoodi, 1098

McBride, S., S2.3, S2.6, S2.7

McCall, D., S3.76

McCarter, J., S3.21

McCarthy, R. L., S3.27, S3.77

McCarty, D., S2.11

McClure, M. A., S3.76

McCorkle, K., S3.77

McCormick, S. P., S3.140

McCotter, S. W., S3.77

McCullough, M., S3.77, S3.85, S3.183

McCutcheon, J., S3.148

McDermott-Kubeczko, M. E., S3.77

McDonald, B. A., 95, 324, 985, S3.23, S3.24, S3.152

McDonald, M. R., 1078, S3.28, S3.29, S3.31, S3.32, S3.77, S3.117, S3.121

McElrone, A. J., S3.43

McFarland, K. A., S1.3, S3.65

McGaughey, R., S3.145

McGee, R., S3.26

McGhee, G. C., S3.3

McGlone, K. S., S3.68

McGrath, J., S3.50

McGrath, M. T., S1.4, S3.78

McGuire, B., S3.182

McHale, L., S3.165

McInroy, J. A., S3.71, S3.132

McKeel, C., S3.143

McKeowen, A., S3.117

McLane, H., 211

McLane, H. L., 1232, S3.63

McLaren, D., S3.53

McMahon, B., S3.36

McMahon, M. B., S3.161

McManus, P. S., S3.129

McMechan, A. J., S3.131, S3.152

McMillan, M., S3.128

McMoran, D., S3.181

McNally, R. R., S3.74, S3.78, S3.173

McNellis, T., S1.5

McOwen, N., S3.136

McPhee, K., S3.40, S3.174

McPherson, B., S3.148

McRoberts, N., 1349, S3.45, S3.61, S3.97, S3.180

McSorley, R., S3.10

McSpadden Gardener, B., S3.30, S3.145

McTavish, C. K., S3.78

Medina, A., S3.44

Medina, R., S3.11

Meekes, E. T., S3.78

Mehl, H. L., S3.79

Mehl, K. M., S3.78, S3.173

Meier, A., S3.79

Meinhardt, S., S3.60

Mekuria, T. A., 548

Melanin: appressoria turgor pressure and, 977

Melcher, U., 1125

Melero-Vara, J. M., 403

Meller Harel, Y., 150

Mello, A. F., S3.79

Melnichukf, F., S3.79

Melo, I. S., 1298

Meloidogyne avirulence protein family: multiple ligand-like motifs in, 879

Meloidogyne spp.: Meloidogyne avirulence protein family, multiple ligand-like motifs in, 879

-M. chitwoodi: nondestructive imaging in planta, 497
-M. chitwoodi resistance: conferred by RNA interference of effector gene Mc16D10L, 1098

-M. hapla: FTA technology for DNA-based analyses, 306

$-M$. incognita: effects on cotton growth and root morphology, 507

- M. javanica: avirulent and $M i$-virulent isolates, responses of tomato genotypes, Israel, 484

Melzer, M., S3.32, S3.79, S3.162

Membrane lipids: effect on plant sensitivity to fengycin, 1036

Menalled, F. D., S3.94, S3.183

Mendoza-Yerbafría, A., S3.34

Meng, Q., S1.3

Meng, R., S3.126

Meng, Y., S3.79, S3.83, S3.132

Menzel, W., S3.146

Mergoum, M., S3.71, S3.165, S3.175

Mert, Z., S3.23

Mertely, J., S3.80

Merten, P. R., S3.30, S3.48

Mesarich, C., S3.154

Mescher, M., S3.151

Mestre, P., 692

Metagenomic data: screening for viruses using eprobe diagnostic nucelic acid assay, 1125

Meyer, F., S2.11

Meyer, S., S3.52

Meyer, S. E., 1306, S3.42

Meyer, S. L., S3.163

Meyer, S. L. F., S3.80

Meyers, K. T., S3.54

Michailides, T., S3.101

Michaud, D., S3.63

Michel, A., S3.165

Michelmore, R., S3.75

Micheloud, N. G., 970

Michler, C. H., S3.54

Midboe, S., S3.3

Mideros, M. F., S3.97

Migita, J., S3.162

Mikaberidze, A., 324

Mila, A., S3.15

Milagres, C. A., S3.97

Milanesi, D. F., S3.22

Milenovic, M. V., S3.64

Miles, L. A., S3.82

Miles, T. D., S3.80, S3.100

Milgroom, M. G., 1221, S3.153

Millas, P. A., S3.80

Miller, C. J., S3.68

Miller, G., S3.91

Miller, G. L., S3.81

Miller, L., S2.8, S3.44

Miller, L. A., 977

Miller, S. A., S3.12, S3.73, S3.115, S3.123, S3.142, S3.156, S3.157

Miller, W. A., S3.81

Miller, Z., S3.183

Miller, Z. J., S3.94, S3.183

Miller-Butler, M., S3.111

Millican, M., S3.81

Millican, M. D., S3.173

Millipede vectors: of grapevine trunk disease pathogens, 1063, cover photo: October

Milosavljevic, A. G., S3.34, S3.120

Milus, E., S3.81

Minsavage, G. V., S2.10, S3.91

Mitcham, E. J., S3.150

Mitchell, T. K., S2.4, S3.68

Mitchum, M. G., 879

MItkowski, N., S1.3

Mitochondrial genes: multiplex assay for genusand species-specific detection of Phytophthora based on, 733
Mitter, N., S3.89, S3.107

Mi-virulent Meloidogyne javanica: responses of tomato genotypes to, 484

Mmbaga, M. T., S3.56, S3.61, S3.100

Mochizuki, T., 1001

Mockler, T., S3.40

Mohamed Nor, N. M., S3.173

Mohammadi, M., S3.184

Moko disease: caused by Ralstonia solanacearum phylotype II strains in Brazil, 1175

Moktan, K., S1.1

Molina, O. I., S3.81

Mollov, D., S3.81, S3.132

Moncayo, L., S3.82

Moncayo-Estrada, R., S3.18

Moncrief, I., S3.82

Monday, T., S3.84

Monfort, S., S3.113

Moniliophthora roreri: in cacao agroforests, effects of shade tree spatial structure on, 275

Monis, J., S3.82

Montana-Lara, J. S., S3.99

Montero-Astúa, M., S3.82

Montesinos, E., 1192

Montgomery, W. S., S3.21

Moon, D., S3.81

Moore, M. J., S3.54, S3.82

Moore, P. P., S3.66

Moore, W., S2.9, S3.2

Moorman, G. W., S2.4, S3.44, S3.66

Moraes, L. A. B., 1298

Moraes, W. B., S3.82, S3.83

Moragrega, C., 1192

Morales-Cruz, A., S3.95

Morawo, T., S3.84

Moreira, L., S3.82

Moreira, W. R., 143, 820, S3.9

Moreno, J. L., S3.7

Morgan, M. J., 749

Morgan, R., 188, S3.127

Morra, M. J., S3.80

Morris, K. A., S2.7

Morrison, E. N., S3.83

Mortensen, C., S3.79

Mosca, S., 851

Mosier, N. J., S3.33

Mosquera, G. M., 436, S3.93

Mostert, L., 1063

Mou, Z., S3.91

Mourelos, C. A., 357

Mourkidou, E., S3.63

Moussart, A., 1138

Moustaid-Moussa, N., S3.20

Mowery, B. K., S3.68

Moya-Elizondo, E. A., S3.83

Moyo, P., 1063

Mtung'e, O. G., S3.32, S3.79, S3.83

$\mathrm{Mu}, \mathrm{W} ., \mathrm{S} 1.4$

Mueller, D., S3.58, S3.159, S3.177

Mukanga, M., S3.57

Mukiyanova, G., S3.5

Müller, V., 1340

Multiplex assay: for genus- and species-specific detection of Phytophthora spp., 733

Munck, I., S1.1

Munck, I. A., S1.4, S3.83

Munitz, T., 1152

Munkvold, G., S3.20

Munkvold, G. P., 1322, S3.29, S3.37, S3.110

Munoz, C. L., S3.84

Munyaneza, J., S3.124

Murillo Williams, A., S3.46, S3.149

Murphy, J. A., S3.13, S3.160

Murphy, J. F., S3.36, S3.84

Murray, S., S3.140 
Murray, T. D., S3.38, S3.76, S3.104, S3.122, S3.185

Musa spp.: silicon-potentiated phenylpropanoid pathway in roots during Fusarium oxysporum $\mathrm{f}$. sp. cubense infection, 597, cover photo: June

Musungu, B., S3.84, S3.139

Mutai, C., S3.45

Mutegi, C., S3.54

Mutegi, C. K., S3.139

Mutiga, S. K., 1221

Mycology: Fusarium graminearum virulence factors and disease severity on soybean, 1201; multiplex assay for genus- and species-specific detection of Phytophthora, 733; Phakopsora pachyrhizi, characterization and quantification in soybean genotypes, 86; Phakopsora pachyrhizi, melanin-independent accumulation of turgor pressure in appressoria, 977

Mycotoxin: contamination in Kenyan maize mills, 1221

Myers, J. R., 786

Myers, K., S3.30

Myers, K. L., S1.4

Myers, R., S3.110

Myresiotis, C., S3.63

Na, F., S3.182

nad9 gene: multiplex assay for detection in

Phytophthora, 733

Naegele, R. P., 479, S3.84

Naghavi, M. R., S3.4

Naidu, R., S3.34

Naidu, R. A., 548

Nakhla, M. K., S3.14, S3.63, S3.88, S3.92, S3.95, S3.130

Nam, S. Y., S3.58

Naranjo, P., S3.33

Narvaez, D., S3.84

Nascimento, K. J. T., 1183

Navarre, D., S3.85

Navarro, S., S3.87

Naveed, K., S3.89

Navi, S. S., S3.85, S3.173

Naz, R., 933

Neal, L. C., S2.1

Nearest percent estimates, 575

Negi, S. S., S3.130

Negron, J., S3.35

Neher, O. T., S3.16, S3.85

Neibling, H., S3.85

Nelson, B., S3.54, S3.138

Nelson, B. D., S3.27, S3.133, S3.138

Nelson, B. D., Jr., S3.27

Nelson, E., S3.29

Nelson, L., S2.8

Nelson, M. E., S3.45

Nelson, R., S3.184

Nelson, R. J., 1221

Nematology: characterization of Pratylenchus thornei resistance in wheat, 174; discrimination of plant-parasitic nematodes from soil communities using ecometagenetics, 749; effects of Meloidogyne incognita and Thielaviopsis basicola on cotton growth and root morphology, 507; FTA technology for DNA-based analyses, 306; Meloidogyne avirulence protein family, multiple ligand-like motifs in, 879; Meloidogyne chitwoodi resistance conferred by RNA interference of effector gene Mc16D10L, 1098; nondestructive imaging of nematodes in planta, 497; responses of tomato genotypes to avirulent and $\mathrm{Mi}$-virulent Meloidogyne javanica isolates, 484

Nester, S. E., S3.17, S3.89

Netland, J., S3.86
Netsu, O., 313

Newberry, E., S3.11

Newcomb, M., S3.56

Newell, M., S3.87

Newman, M. A., S2.5, S3.56

Newman, S., S2.10

Next generation sequencing data: screening for viruses using e-probe diagnostic nucelic acid assay, 1125

Ngo Bieng, M. A., 275

Ni, X., S3.126

Niblack, T. L., S3.68, S3.72, S3.110

Nichols, A., S3.76

Nichols, R., S3.73

Nicot, P. C., 859

Nicotiana spp.

-N. occidentalis: antagonistic interactions of

'Candidatus Phytoplasma mali' strains in, 453

-N. tabacum: abscisic acid-cytokinin

antagonism modulates Pseudomonas syringae resistance in, 1283

Nikolaeva, E., S3.61

Nikolaeva, O. V., 786

Nischwitz, C., S3.77, S3.85, S3.183

Nishiguchi, M., S3.85

Nita, M., 692, S3.85, S3.162

Nix, K. A., S3.30, S3.48

Njuguna, J., S3.45

Njus, Z. L., S3.29

Noe, J. P., S3.183

Noel, Z. A., S1.5

Noh, J., S3.58

Nordskog, B., S3.86

Noreskal, M., 586

Norland, J. E., S3.138

Norman, D. J., S3.16

Normantovich, M., S3.8, S3.91

Noveroske, A. J., S3.173

Novy, R., S3.124

Ntasiou, P., S3.63

Nunes, W. M., S3.18, S3.86

Nurseries: Phytophthora community structure in

Oregon nurseries, 1052

Nutter, F. W., S3.37

Nutter, F. W. Jr., 365

Obradovic, A., S3.64

OBrian, G., S3.139

O'Brien, G., S3.84

Ochoa, J. B., 379

Ochoa, O., S3.75

Ochoa-Corona, F. M., S3.34

Ockey, S., S3.51

Ockey, S. C., S3.54

Odom, J., S3.86

Odom, J. L., S3.174

O'Donnell, K., S3.111, S3.148

Ogunjobi, A. A., S3.62

O'Hara, C., S2.5

Ohki, S. T., 1001

Ohki, T., 313

Ojiambo, P., S3.68, S3.118, S3.130

O'Keeffe, T., S3.89

'Okitsu' mandarin: canker disease resistance, role of leaf features in, 970

Okmen, B., S3.154

Okpako, E., S3.35

Okubara, P. A., 683

Olarte, R. A., 75

Olaya, G., S3.86

Oldach, K. H., 174

Oliari, L. S., S3.109

Olivares-Garcia, C., S3.153

Oliveira, M. S., S3.86

Oliveira, S. C., S3.23
Oliver, C., S3.85, S3.162

Oliver, R., 1264

Olivera, P., S3.56

Olotuah, O. F., S3.87

Olsen, K. M., S3.70, S3.71

Olsen, N., S3.180

Olsen, N. L., S3.104

Olsen, R., S3.146

Olson, J., S3.34

Olson, S. M., S3.64

Olson, T. N., S3.61

Olson, W., 1163

Omarov, R., S3.5

Ommattoiulus moreleti: vector of grapevine trunk

diseases, 1063, cover photo: October

O’Neal, E. S., 282, S3.148, S3.162

Ong, K., S2.2, S2.3, S2.7, S3.45

Onion thrips, 812

Onofre, R. B., S3.8

Onuki, M., 313

Ophel-Keller, K., S3.91

Ordonez, M., S3.62

Oregon grape, 1208

Orlovskis, Z., S3.150

Orquera, G., S2.8

Ortiz-Ribbing, L. M., S3.87

Oryza sativa: comparative analysis of rice seed bacterial pathogens, 436; detection of

Xanthomonas oryzae pv. oryzae and $X$. oryzae pv. oryzicola with a padlock probe-based assay, 1130; Pi-ta gene, effectiveness and durability in China, 762; synergism between Southern rice black-streaked dwarf virus and Rice ragged stunt virus, 794

Osborne, L. E., S3.58

O’Shaugnessy, S., S3.109

O'Shea, P., S3.127

Osorio, L. F., 67

Osorno, J. M., S3.172

Ospina, Y., S3.93

Osterbauer, N., S3.87

Osti, S., S3.87

Ostrander, J., S3.174

Ostry, M. E., S3.54, S3.82

Osuagwu, A., S3.35

Osunga Buyu, H. A., S3.87

Oßwald, W., S3.18

Otero-Colina, G., S3.101

Ottesen, A., S3.55, S3.87

Otto-Hanson, L., S3.8, S3.88

Otto-Hanson, L. K., S3.106, S3.175

Oudemans, P. V., S1.6

Ouedraogo, L., 520

Owens, K. J., S3.88, S3.92

Ownley, B. H., S3.20, S3.88, S3.108

Ozalvo, R., 484

Ozias-Akins, P., S2.7

Pace, M., S3.85

Padasht Dehkaei, F., S3.4

Padlock probe-based assay: detection of Xanthomonas oryzae pv. oryzae and X. oryzae pv. oryzicola in rice, 1130

Paenibacillus elgii MM-B22: induction in cucumber of priming-mediated systemic resistance against Colletotrichum orbiculare, 834

Paetzold, L., 126, S3.88

Pagliaccia, D., 188

Palm, M. E., S3.88

Palmateer, A. J., S3.16, S3.115

Palmer, C. L., S3.17, S3.89, S3.146, S3.147

Palumbo, J., S3.89

Pan, Q., S3.89

Pan, S., 188 
Pandey, S., S3.29

Pang, Z., 269

Panthee, D., S3.109

- P. agglomerans: transmission by onion thrips, 812

-P. ananatis: transmission by onion thrips, 812

Pantoea spp.

Paolinelli-Alfonso, M., S3.183

Pappu, H., S3.89, S3.103, S3.106, S3.107, S3.120

Pappu, H. R., 202

Parada Rojas, C. H., S3.89

Pardo, J. M., S3.7

Paret, M. L., S3.11, S3.64, S3.114

Parikh, L., S3.61

Park, C. M., S3.60

Park, J. H., S3.60, S3.107, S3.148

Park, J. J., S3.60, S3.64

Park, Y. L., S3.163

Parke, J. L., 1052, S3.142

Parker, J. K., 15

Parker, M., S3.51

Parkunan, V., S3.55, S3.92

Parra, C. C., S3.63

Partelli, F. L., S3.109

Parthogenesis-related proteins: expression in wheat leaves pre-treated against leaf rust with plant extracts, 933

Pasche, J., S3.86, S3.172, S3.174

Pasche, J. S., 40, S3.49, S3.170

Paschen, M., S3.77

Pascholati, S. F., S3.18

Pastor-Corrales, M. A., S3.90

Patel, J. S., 40, S3.90

Patev, S., S3.90

Patricia, B., 484

Pattison, J. A., 67

Paul, C., 86

Paul, P. A., 702, S3.102, S3.110

Paulitz, T. C., 683

Paveley, N., 1264

Pavon, C., S3.10

Pawlowski, M., 843, S3.90

Payne, G., S3.84, S3.139

Payne, G. A., S3.68

Payton, M. E., S3.47

PCA-producing Pseudomonas sp. LBUM223:

long-term induction of defense gene expression

in potato, 926

Peach gummosis: carbohydrate metabolism

changes in Prunus persica infected with

Lasiodiplodia therobromae, 445

Pearce, T. L., S3.90, S3.105

Pearson, C. A. S., S3.144

Pedersen, D. K., S3.135

Pedersen, P., S3.86

Pedrozo, R., S3.90

Peever, T. L., S3.60, S3.158

Pelz-Stelinski, K., S3.141

Peng, G., S3.40

Peralta, C., S3.123

Pereira, J., S3.91

Pereira, L. F., 34

Pereira Silva, K. J., S3.91

Peres, N., S3.41, S3.91

Peres, N. A., 67, 396, 954, S3.8, S3.80, S3.86

Pereyra, S. A., S3.91

Perez, C. A., S3.6

Perez, C. E., S3.97

Perez, W., 624

Perez-Garcia, M., S3.34

Perez-Hernandez, O., S3.91, S3.167, S3.174

Perkins, A., S3.140

Perl-Treves, R., S3.8, S3.91

Perna, N. T., S3.101

Peronospora spp.
-P. effusa: coupling spore traps and $\mathrm{qPCR}$ assays for detection of, 1349

-P. schachtii: coupling spore traps and $\mathrm{qPCR}$ assays for detection of, 1349

- P. sparsa: in boysenberry, disease risk prediction model, 50

- P. variabilis: in quinoa seed, molecular

detection and phylogeny, 379

Perring, T. M., S3.95

Perry, K. L., 211, 1232, S3.63

Pesic, Z., S3.6

Pesic-Vanesbroeck, Z., S2.1

Peters, R., S3.57

Peterson, D., S3.73

Pethybridge, S. J., S3.90, S3.91, S3.105

Petkar, A., S3.55, S3.92

Petri disease: arthropod vectors, 1063

Pettengill, J., S3.55

Petunia: distribution of Potato spindle tuber viroid in floral organs, 964

Pfaf-Dolovac, E. L., S3.34, S3.120

Pfender, W., S3.40

Pfeufer, E., S1.5, S3.92

Pfeufer, E. E., S3.142

Phaeomoniella chlamydospora: arthropod vectors, 1063

Phakopsora pachyrhizi: characterization and quantification in soybean genotypes, 86; melanin-independent accumulation of turgor pressure in appressoria, 977

Pham, J., S2.6

Phaseolus vulgaris: Bean common mosaic virusinduced temperature-insensitive necrosis in $I$ gene-bearing plants, 1251; Bean mosaic virus infection, cover photo: November; interaction of resistance factors with genetic determinants of Bean common mosaic virus, 786

Phat, P., S3.57

Phellinus spp.: on citrus in Italy, characterization of, 851

Phenazxine-1-carboylic acid, 926

Phenylpropanoid pathway: potentiation by silicon in banana roots during Fusarium oxysporum $\mathrm{f}$. sp. cubense infection, 597

Phibbs, A., S3.81

Phillips, T., S3.51

Phylloxera galls: of Vitis riparia, with downy mildew, cover photo: July

Phytophthora spp.: community structure in Oregon nurseries, 1052; multiplex assay for genus- and species-specific detection, 733

-P. capsici: competition between pyrimorphsensitive and pyrimorph-resistant isolates, 269; evolution of an experimental population in the field, 1107

-P. capsici resistance: mapping in pepper, 479

$-P$. infestans resistance: in potato genotypes adapted to tropical highlands, 624

Picton, D. D., S3.92, S3.130

Pierce, B., S3.92

Pierce, M. L., 1088

Pierce's disease of grapevine. See Xylella fastidiosa

Pierson, E., S3.64

Pierson, E. A., S3.68, S3.125

Pierson, L. S., III, S3.125

Pieterse, C. M. J., 926

Pilkington, S., S3.105

Pilson, D., S3.110

Pinus flexilis: white pine blister rust resistance,

163, cover photo: February

Piotto, F., S3.121

Piqueras, C. M., S3.92

$\mathrm{Pi}$-ta gene: effectiveness and durability in China, 762
Pitino, M., S3.92

Plant extracts: pretreatment of wheat leaves against leaf rust, 933

Plasencia, J., S3.93

Plasmodiophora brassicae: genetic structure, effects of oilseed rape cropping and geographic origin on, 532; role of primary and secondary infection in host response to, 1078

Plasmopara viticola: geographic distribution of cryptic species, eastern North America, 692

Plesko, I. M., S3.117

Ploetz, J., S3.148

Ploetz, R. C., S3.21, S3.148

Plum bark necrosis stem pitting-associated virus: characterization and development of a broadspectrum detection assay, 660

Plum pox virus: e-probe diagnostic nucleic acid assay, 1125

Podosphaera aphanis: ontogenic resistance in strawberry, 954, cover photo: September

Poh, T., S3.42

Pol, C., S3.183

Polashock, J., S3.117, S3.129

Pollen transmission: of Asparagus virus 2, 1001

Pollok, J., S3.93

Polymerase chain reaction (PCR) assays: RT$\mathrm{PCR} /$ macroarray multiplex platform to profile viral infections in grapevine, 211; TaqMan PCR assay for Synchytrium endobioticum in soil, 422; Verticillium dahliae race 2 -specific, 779. See also Quantitative polymerase chain reaction assays; Real-time PCR assays

Poole, G. H., S3.16

Pooler, M., S3.146

Popko, J. T., S1.5

Poplawsky, A. R., 786, 1251, S3.152

Population biology: asexual reproduction of Puccinia striiformis f. sp. tritici in the Pacific Northwest, 1208; characterization of Fusarium oxysporum species complex isolates from soybean roots, 1329; Colletotrichum acutatum, genetic structure, Brazil, 769; evolution of an experimental population of Phytophthora capsici in the field, 1107; fitness attributes of Fusarium graminearum with 3-ADON or 15ADON trichothecene genotypes, 513; Fusarium oxysporum from sugar beet, multilocus analysis, 886; Magnaporthe oryzae population structure and pathotype diversity, Brazil, 95;

Plasmodiophora brassicae genetic structure, effects of oilseed rape cropping and geographic origin on, 532; rice $P i$-ta gene, effectiveness and durability in China, 762; Verticillium dahliae, clonal expansion in lettuce, 641; Verticillium dahliae, molecular relationship between vegetative compatibility groups in, 650; Xanthomonas oryzae pv. oryzicola population analysis, Mali and Burkina Faso, 520

Porazinska, D. L., 749

Porch, T. G., S3.90

Porchas, M., S3.75

Porter, L., S3.174

Portuguese millipede, cover photo: October; vector of grapevine trunk diseases, 1063

Postharvest pathology and mycotoxins: lipoxygenase activation in peanut seed cultivars in response to Aspergillus parasiticus colonization, 1340; mycotoxin contamination in Kenyan maize mills, 1221

Potato spindle tuber viroid: distribution in petunia floral organs, 964

Potnis, N., S2.10

Potter, J. L., 211

Poudel, R., S3.93

Poussier, S., 520 
Pozniak, C., 1322

Pozo, M. J., S3.5

Prabhu, A. S., S3.47

Pradhan, S., S3.44

Pradhan Shrestha, S., S2.8

Prado, G. A., 436, S3.93

Pratylenchus spp.

-P. neglectus: discrimination from soil

communities using ecometagenetics, 749

$-P$. penetrans: nondestructive imaging in planta, 497

-P. thornei: discrimination from soil

communities using ecometagenetics, 749

-P. thornei resistance: in wheat, characterization of, 174

Prendeville, H. R., S3.110

Prenni, J. E., S3.99, S3.128

Price, J. A., S2.8, S3.158

Price, P., S2.1

Price, P. P., S3.5

Priming-mediated systemic resistance: induction in cucumber against Colletotrichum orbiculare, 834

Pringle, H., S3.128

Prior, P., 1175

Pritsch, C., 715

Proaño, F., S2.4, S3.44

Probst, C., S3.45, S3.139

Proctor, R. H., S3.140

Prophete, E., S3.90

Prospero, S., 918

Pruner, R., S3.111

Prunus persica: carbohydrate metabolism changes

with infection by Lasiodiplodia therobromae, 445

Psammotettix alienus: localization and

distribution of Wheat dwarf virus in, 897

Pscheidt, J., S3.181

Pscheidt, J. W., S3.48, S3.135

Pseudomonas spp.

-P. azotoformans GC-B19: induction in cucumber of priming-mediated systemic resistance against Colletotrichum orbiculare, 834

-P. chlororaphis subsp. aurantiaca: strain Pcho10 as a potential biocontrol agent for Fusarium graminearum, 1289

-P. fluorescens HC1-07: biological control of wheat root diseases, 248

$-P$. sp. LBUM233: long-term induction of defense gene expression in potato, 926

-P. syringae complex: definition of plantpathogenic genomospecies in, 1274

- P. syringae resistance: abscisic acid-cytokinin antagonism modulates in tobacco, 1283

Pterostilbene: inhibitory effects on in vitro growth of soybean pathogens, 843

Pu, L., 196, S3.65, S3.93

Puccinia spp.

-P. striiformis: Yr36 gene confers partial resistance in wheat, 871

-P. striiformis $\mathrm{f}$. sp. tritici: asexual reproduction in the Pacific Northwest, 1208

-P. striiformis resistance: race specificity in wheat, 1042

-P. triticina: pathogenesis-related protein expression in wheat leaves pre-treated against with plant extracts, 933

-P. triticina resistance: in durum wheat, genetic analysis of, 1322; in wheat breeding lines, molecular mapping, 865

Pugliese, M., S3.48

Puig, M., 1192

Puri, K., S3.4

Purple marsh crab: tripartite interactions in salt marsh dieback, 1070
Purvis, M. D., S3.55

Pyle, J. D., S3.114

Pyramiding $B$ genes: bacterial blight resistance in cotton, 1088

Pyricularia oryzae: effects on leaf gas exchange and chlorophyll $a$ fluorescence in silicon inoculated wheat, 143; identification of a hidden resistance gene in tetraploid wheat, 634; impairment of wheat leaf photosynthesis, 34

Pyrimorph resistance, 269

Pyrus communis: postinfection activity of synthetic antimicrobial peptides against Stemphylium vesicarium, 1192

Qi, X., S3.70, S3.71

Qian, G., 224

Qiao, Y., 188

Qin, Z., 269

Qiu, W., 539

Quantitative polymerase chain reaction (qPCR) assays: assay for Cercospora kikuchii in soybean leaves, 1118; coupling with spore traps to detect downy mildews of spinach and beet, 1349. See also Real-time PCR assays

Quesada, L., S3.105

Quesada-Ocampo, L. M., S3.84, S3.89, S3.123, S3.130

Quinone outside inhibitor (QoI) resistance: in

Botrytis cinerea, 347

Quintero-Ferrer, A., S3.183

Quito-Avila, D. F., S3.94, S3.141

Radmer, L., S3.94

Radovich, T., S3.162

Radwan, O., S3.94

Rafoss, T., S3.86

Rahman, A., 614

Rahman, M., S3.163

Raid, R., S3.100

Raid, R. N., S3.94

Raikhy, G., S3.106, S3.120

Rajakaruna, P., S2.2

Rajamäki, M. L., S3.151

Rajashekara, G., S3.123

Rakotondrafara, A., S3.81, S3.94, S3.166, S3.174, S3.177

Rallo, L., S3.120

Ralstonia solanacearum: diversity in French

Guiana, 586; phylotype II, Moko disease-

causing strains in Brazil, 1175

Ramadugu, C., 257

Ramirez-Suero, M., 1021

Ramming, D., S3.152

Ramos, L., S1.5

Ramos Molina, L. M., S3.24

Ramos-Pena, I. M., S3.99

Ranabhat, N. B., S3.94, S3.183

Randall, J. J., S3.95, S3.184

Randoux, B., 293

Ranjan, A., S3.95

Ransom, J., S3.171

Rapicavoli, J. N., S3.95

Raruang, Y., S3.95

Rascoe, J. E., S3.95

Rashed, A., 126, S3.88

Rasheed, M., S3.124

Rashid, K. Y., S3.36, S3.95, S3.96

Rasmussen, J. B., S3.58

Rast, T. J., S3.179

Rath, M., S3.46

Rav-David, D., 150

Ravindran, A., S3.68

Ravn, H. P., S3.116

Ray, S. L., S3.61

Raymer, P., S3.75
Razi, M. F., 257

Real-time PCR assays: for Aphanomyces euteiches, 1138; for 'Candidatus Liberibacter solanacearum', 804. See also Quantitative polymerase chain reaction

Rebollar-Alviter, A., S3.18

Redhead, S. A., 422

Redinbaugh, M. G., 1360

Reed, D., S3.93

Reed, E., S3.87

Reed, S., S3.96, S3.148

Reeser, P., S3.153

Reges, J. T. A., S3.23

Rehard, D. G., S3.170

Reich, J., S3.96

Reignault, P., 293

Reisch, B., S3.152

Reiter, M. S., S3.98

Ren, C., S3.96

Resende, R. S., S3.97

Restrepo, S., S3.97, S3.99

Resveratrol: inhibitory effects on in vitro growth of soybean pathogens, 843

Reuter-Carlson, U., S3.135

Revell, J. M., S3.17, S3.89

Reverse transcription-PCR (RT-PCR): RT-

$\mathrm{PCR} /$ macroarray multiplex platform to profile viral infections in grapevine, 211

Reyes, P. E., S3.91

Reyes Chin-Wo, S., 479

Reyes Gaige, A., S3.97

Reynolds, G. J., S3.97

Rezende, D., S3.97

Rezende, J., S3.97

Rhizoctonia spp.: agroecological factors correlated to soil DNA concentrations, Washington, 683

-R. oryzae: agroecological factors correlated to soil DNA concentrations, Washington, 683; increasing spore susceptibility to heat stress, 240

$-R$. solani: inhibitory effects of stilbenes on in vitro growth, 843

$-R$. solani AG8: agroecological factors correlated to soil DNA concentrations, Washington, 683; biological control by Pseudomonas fluorescens HC1-07, 248

Rhoads, B., S3.85, S3.183

Riano-Pachon, D., S3.97

Riaz, K., S3.50

Ribero, S., 422

Rice ragged stunt virus: synergism with Southern rice black-streaked dwarf virus, 794; virusplant-vector interactions with Southern rice black-streaked dwarf virus co-infection, 196 Richard-Cervera, S., 692

Richards, J., S3.54

Rideout, S. L., S3.98

Riely, B. K., S3.122

Rigling, D., 918

Riley, D. G., 202

Riley, I. T., 174

Riley, T. D., S3.98

Rimbaud, L., S3.98

Rincon, A. M., S3.97

Rios, J. A., 34

Rioux, R., S3.98

Rista, L. M., 970

Ristaino, J. B., S3.104

Ritchie, L., S3.64

Rivera, C. M., S3.87

Rivera, V. V., S3.106

Rivera, Y., S1.5, S3.74, S3.98, S3.102, S3.146, S3.163

Rivière, P., 586

Rizzo, D. M., S3.9, S3.50 
$\operatorname{RmgTd}(t)$ gene: in tetraploid wheat, 634 RNA interference (RNAi): of effector gene Mc16D10L confers resistance to Meloidogyne chitwoodi, 1098

Robbins, R. T., S2.6, S3.59

Roberson, S., S2.8

Roberts, M. J., S2.8

Roberts, P. A., S3.126

Roberts, P. D., S3.149

Roberts, R., S3.94, S3.174, S3.177

Roberts, T. H., 933

Robertson, A., S3.76, S3.166

Robertson, A. E., 365, S3.2, S3.37

Robertson, C. L., 1118, S2.2, S3.109

Robideau, G. P., S3.14, S3.98

Robinson, R. R., S3.99

Robison, F. M., S3.99

Roccotelli, A., 851

Rocheleau, H., 422

Rodrigues, F. A., 34, 143, 597, 820, 1183, S3.9, S3.14, S3.97, S3.99

Rodríguez-Alvarado, G., S3.126

Rodriguez-Bocanegra, M. X., S3.99

Rodríguez-Decuadro, S., 715

Rodriguez-Saona, L., S3.148

Roets, F., 1063

Rogers, S. T., S3.172

Roh, J., S2.5

Rohila, J. S., S3.2, S3.5

Roitsch, T., 1283

Rojas, A., S2.10, S3.40, S3.100, S3.127

Rojas, L. A., S3.176

Rollan, C., S3.11

Rollemberg, C. L., 1175

Rollins, J., S3.175

Rollins, J. A., S3.126

Rolshausen, P., S3.133

Rolshoven, J., S3.125

Romberg, M., S3.31

Ronco, B. L., S3.11

Roose, M., 257

Roper, C., S3.21, S3.133

Roper, M. C., S3.95, S3.130

Rosas, J. C., S3.90

Roselló, M., 804

Rosenberger, D. A., S1.6

Rosenberger, S. A., S3.100

Rosenzweig, N., S3.55, S3.100, S3.113, S3.175, S3.176

Roseshclin, R. A., 970

Rosli, H. B., S3.100

Rosskopf, E. N., S3.52

Rotenberg, D., S3.11, S3.151, S3.158

Rothrock, C. S., 507, S2.9, S2.10, S2.11, S3.113, S3.121

Rotich, E., S3.100

Rott, P. C., S3.100

Rouf Mian, M. A., S3.165

Rounsley, S., S3.132

Rouse, D., S3.14, S3.42, S3.59, S3.169

Rouse, D. I., S3.45

Rouse, M., S3.19, S3.157

Rouse, M. N., S3.60

Rouxel, M., 692

Rowland, O., S3.123

Roy, A., S3.101

Ruark, M. D., S3.167

Rubio, I., S3.101

Rubio, L., 108

Rubus spp.: downy mildew disease risk prediction model for boysenberry, 50; fludioxonil resistance in Botrytis cinerea isolates, 724; ipro-

dione resistance in Botrytis cinerea isolates, 396

Rugh, A. L., S1.6

Rugman-Jones, P. F., S3.182
Ruhl, G., S3.81

Rupe, J., S3.28, S3.69

Rupe, J. C., S2.2, S2.9, S2.10, S2.11, S3.121, S3.134

Rupprecht, S. M., S3.80

Rush, C. M., 126, S2.8, S3.88, S3.109, S3.131, S3.158

Russell, P., S3.136

Rutter, W. B., 879

Ruz, L., 1192

Ryšánek, P., 306

Sabanadzovic, S., S2.9, S3.2, S3.101, S3.106, S3.113

Saccharum spp.: Aspergillus parasiticus communities, Texas, 462

Sadek, M. E., S3.106

Sadof, C., S3.159

Sahi, S. T., S3.9, S3.50

Saied, N., S3.74, S3.98

Sain, L., S3.44

Saint-Jean, S., S3.46

Saito, S., S3.101

Sakai, J., 313

Sakalidis, M. L., S3.101

Sakuno, C., S3.121

Salas, B., S3.102

Saleem, M. J., S3.50

Salgado, J. D., S3.102

Salgado-Salazar, C., S3.98, S3.102

Salicylic acid-mediated defense response: in perennial ryegrass against Magnaporthe oryzae, 614

Salt marsh dieback: tripartite interactions in, 1070

Samac, D., S3.102, S3.103

Samac, D. A., S3.23, S3.72, S3.128

Sambaraju, K. R., S1.6

Sambugaro, R., S3.109

Sanchez, I., S3.44

Sanchez, L., S3.176

Sanchez Perez, A., S3.103

Sanchez-Rangel, D., S3.93

Sanders, F. H., S3.103

Sanders, H., 232

Sang, H., S1.2, S1.3, S1.6

Sang, M. K., 834

Sanjuán, S., 804

Sankaran, S., S3.103

Sanogo, S., S3.184

Santa Maria, A., S3.175

Santamaria, L., S3.13, S3.103

Santos, B. M., S2.5

Santos, G. R., S3.20

Santos, L. A., 1175

Sanzani, S. M., 851

Saparrat, M. C., S3.11

Sapkota, S., S3.175

Saponari, M., S3.133

Sarmis, G., S3.62

Sarra, S., 520

Sasaya, T., 313

Sattler, S. E., S3.43, S3.169

Saunders, S., S3.42

Savary, S., S3.104, S3.150

Savelle, A., S3.104, S3.130

Saville, B. J., S3.26, S3.35, S3.83

Sawchyn, K., S3.17

Sazib, B., S3.156

Scanlon, K., S3.104

Schaeffer-Reiss, C., 1021

Schafer, R., S3.168

Schäfer, W., 1201

Schanbel, G., 396

Schauer, A., S3.13, S3.103

Scheidecker, D., S3.81

Schena, L., 851
Scherm, H., S3.130

Scheuring, D., S3.68

Schilder, A., 692, S3.117

Schisler, D. A., S3.104

Schjøll, A. F., S3.86

Schlatter, D., S3.8

Schlund, S. A., S3.66

Schmelz, E. A., S3.139

Schmid, C. J., S3.13, S3.160

Schmidt, C., S3.166

Schmidt, L. S., S3.20, S3.104

Schmidt, S., S3.105

Schnabel, G., 724

Schneider, A., 240

Schneider, B., 453

Schneider, D. J., S3.104

Schneider, R., S2.1, S3.5

Schneider, R. W., 1118, S2.2, S3.109, S3.154

Schneider, W., S3.104, S3.114

Schneider, W. L., 1125, S3.76, S3.101

Schoelz, J., 539

Schoettle, A. W., 163

Scholthof, K. B. G., S3.114

Schroeder, B., S3.77

Schroeder, B. K., S3.76, S3.104

Schroeder, K. L., 683

Schulte-Geldermann, E., S3.51

Schwartz, H., S3.116

Schwartz, H. F., S3.99

Schwebs, S., S3.43

Schweigkofler, W., S3.105

Sciumbato, G., S3.128

Sclerotinia sclerotiorum: inhibitory effects of stilbenes on in vitro growth, 843

Scoglio, C., S3.51

Scortichini, M., 1274

Scott, J. B., S3.90, S3.105

Scraber, S., S3.103

Scruggs, A., S3.105

Scully, B., S2.2, S3.132

$s d h \mathrm{~B}$ gene mutations: fungicide resistance in

Botrytis cinerea and, 347

Sechler, A. E., S3.104

Secor, G., S3.13, S3.36, S3.106

See, D., S3.182

Seem, R. C., 954

Seemüller, E., 453

Segovia, V., 871

Seidl Johnson, A., S3.42

Sella, L., 1201

Selph, A., S2.9

Senghor, L., S3.139

Sentelhas, P. C., S3.28

Seo, J.-K., 993

Seo, Y.-W., S3.108

Sesarma reticulatum: tripartite interactions in salt marsh dieback, 1070

Sessoms, F. J., S3.106, S3.175

Sétamou, M., S3.63, S3.64

Sewake, K., S3.110

Seybold, S. J., S3.135

Shaaban, M., S3.50

Shabana, Y. M., S3.106

Shade tree spatial structure: effects on frosty pod rot in cacao agroforests, 275

Shah, D. A., 702

Shahid, M. S., S3.106

Shamoun, S. F., S3.50, S3.106

Shan, K., S3.125

Shanmugiah, R., S3.106, S3.107

Shao, J., 269

Shapiro-Ilan, D. I., S3.16

Sharanowski, B. J., S3.7

Sharma, K., 1078

Sharma, S., S3.105 
Sharon, A., S3.38

Sharp, K. J., S3.170

Shaver, J. B., S3.107

Shay, R. N., S3.169

Shea-Andersh, M. A., S3.104

Shen, W., S3.131

Shepard, N. R., S1.4

Shepherd, R. W., S3.175

Sherwood, P., S3.107

Sheveleva, E. V., S3.40

Shew, B., S3.72

Shew, D., S3.77

Shew, H. D., S3.62

Shi, J., 188

Shi, S., S3.132

Shiel, P. J., S3.130

Shier, W. T., S3.1

Shim, C. K., S3.60, S3.107

Shim, W. B., S3.162

Shimura, H., 1001

Shin, D. B., S2.5

Shin, G.-H., S3.108

Shin, H. Y., S3.64

Shin, K., S3.148

Shine, J., S3.100

Shiraishi, T., S3.108, S3.119

Shirako, Y., 313

Shishkoff, N., S3.147, S3.163

Shiu, P. K. T., S3.170

Short, D. P. G., 282, 641, 779

Showmaker, K., S3.73

Shrestha, B. K., S3.108

Shrestha, S., S3.108

Shrestha, U., S3.31, S3.108

Shtienberg, D., S3.149

Shu, X., S3.139

Shugart, H. J., S3.95

Sikkens, R. B., S3.36, S3.108

Sikorski, J., 1152

Silicon: effect on leaf gas exchange and chlorophyll $a$ fluorescence in Pyricularia oryzae infected wheat, 143; effect on soybean resistance to Cercospora sojina, 1183; potentiation of the phenylpropanoid pathway in banana roots during Fusarium oxysporum f. sp. cubense infection, 597, cover photo: June

Silva, A. M. F., 1175

Silva, D. D., S3.66, S3.112

Silva, E., S3.14

Silva, E. C., S3.109

Silva, F. F., S3.82

Silva, F. N., S3.112

Silva, L. J., 1298

Silva, M. B., S3.109

Silva, P., 715

Silva-Junior, G. J., 769

Silva-Lobo, V. L., S3.47, S3.109

Silva-Rojas, H. V., S3.18

Silverman, E. J., S3.109

Simmons, A. R., S3.109

Simmons, H. E., S3.110

Simon, A. C. M., S3.110

Simons, K., S3.49, S3.170, S3.172

Sims, J., S3.139

Singh, P., S3.110

Singh, P. K., S3.2, S3.165

Singh, R., S2.9

Singh, R. A., S2.5

Singh, R. K., S3.110

Single nucleotide polymorphisms (SNPs): spatial distribution of SNPs related to fungicide resistance, 604

Sinn, J., S1.5

Sipes, B., S3.110, S3.123

Sivakumar, S. N., S3.111
Skog, T. E., S3.86

Skory, C. D., 240

Slack, S., S3.111, S3.175

Slininger, P. J., S3.104

Small, I., S1.2

Small, I. M., S3.30, S3.149

Smallwood, E. L., S3.161

Smart, C. D., 1107, S1.1, S1.4, S3.115, S3.149

Smart, T. B., S3.111

Smirnova, E., S3.81

Smith, A., S3.112

Smith, B. J., S2.4, S3.111

Smith, D., S3.111, S3.167

Smith, D. L., S3.58, S3.95, S3.130, S3.171

Smith, D. S., 422

Smith, J., S3.35, S3.111

Smith, J. A., S3.148

Smith, K. M., S3.163

Smith, M. J., S3.8, S3.113

Smith, P., 396

Smith, S., 232, S2.3, S3.118

Snelling, J., S3.53

Sniezko, R. A., 163

Soares, J. M., S3.112

Sobolev, V. S., 75

Soby, S., S3.112

Soil fumigation: synergistic effect with

Clonostachys rosea against cucumber wilt, 1314 Solanum spp.

-S. lycopersicon: Botrytis cinerea genotypes in tomato greenhouse populations, 859 ; gray mold resistance induced by benzothiadiazole and

Trichoderma harzianumi T39, 150; responses to avirulent and Mi-virulent Meloidogyne javanica isolates, Israel, 484

-S. tuberosum: late blight resistance in genotypes adapted to tropical highlands, 624; long-term induction of defense gene expression by Pseudomonas sp. LBUM233 and

Streptomyces scabies, 926; Meloidogyne chitwoodi resistance conferred by RNA interference of effector gene Mc16D10L, 1098; physiological responses to 'Candidatus

Liberibacter solanacearum' infection, 126

Soliman, M. S., S3.37

Somohano, P., S3.168

Somohano, P. M., S3.55

Song, J., S3.108

Sørensen, C. K., 1042

Sorensen, R. B., 75

Sorghum halepense: viruses in maize and

Johnsongrass in southern Ohio, 1360

Soto-Arias, J. P., S3.112

Soubeyrand, S., S3.98

South, M. S., S3.21

Southern rice black-streaked dwarf virus: synergism with Rice ragged stunt virus, 794; virus-plant-vector interactions with Rice ragged stunt virus co-infection, 196

Souza, A. G., S3.66, S3.112

Souza, A. G. C., S3.20, S3.82, S3.83

Souza, A. N., S3.112

Souza, A. P., 769

Souza, E. B., 1175

Souza, W. R., 1298

Spadaro, D., S3.48

Spagnolo, A., 1021

Spartina alterniflora: tripartite interactions in salt marsh dieback, 1070

Spector, T., S3.111

Spence, D., S3.148

Spence, K. L., S3.26, S3.35

Spiceland, D., S3.113

Spider vectors: of grapevine trunk disease

pathogens, 1063
Spies, C. F., S3.113

Spinacia oleracea: coupling spore traps and qPCR assays to detect downy mildew of, 1349; Verticillium dahliae race 2 strains in seed lots, 779

Spiroplasma citri: novel diagnosis for citrus stubborn disease, 188

Spitzer, W. E., S2.9

Spolti, P., 513

Spore traps: coupling with qPCR assays to detect downy mildews of spinach and beet, 1349

Spraker, J. E., S3.176

Spröer, C., 1152

Spurlock, T. N., S3.113

Srinivasan, R., 202, 812, S3.151

Staack, D., S2.5

Stack, J., S3.82, S3.97

Stack, J. P., S3.9, S3.21

Stahl, S. L., S3.113

Stamler, R., S3.95

Stamler, R. S., S3.184

Standish, J., S3.19, S3.113

Standish, J. R., S2.9

Stanghellini, M., S3.184

Stanton, J. L., S3.113

Stanton, T., S3.154

Stedtfeld, R., S3.159

Steere, L., S3.100, S3.113, S3.175, S3.176

Steffenson, B., S3.23

Steger, A., S2.2, S2.11, S3.134

Steinke, K., S3.100, S3.175

Steketee, C. J., S3.75

Stelinski, L. L., S3.151

Stemphylium vesicarium: postinfection activity of synthetic antimicrobial peptides against in pear, 1192

Stensvand, A., 954

Stephens, J. Y., S3.110

Stephenson, A. G., S3.110

Stephenson, R. C., S3.101

Stetina, T. J., S2.9

Stevenson, K. L., S3.104

Stewart, B. R., S3.122

Stewart, C. L., S3.114

Stewart, E., S3.152

Stewart, E. L., 985

Stewart, J. E., S3.114, S3.153

Stewart, L. R., 1360

Stilbenes: inhibitory effects on in vitro growth of soybean pathogens, 843

Stobbe, A. H., 1125

Stocks, S. D., S3.77

Stone, A., S3.101, S3.114

Stoneman, W., S3.145

Stouthamer, R., S3.182

Stowell, L., S3.184

Strauss, J. A., S3.15

Strauss, S., S3.114

Strayer, A., S3.114

Strehlow, B., 532

Strelkov, S. E., S3.22, S3.39, S3.42, S3.53, S3.136, S3.138

Streptomyces spp.

$-S$. araujoniae: biocontrol for Botrytis cinerea, 1298, cover photo: December

-S. scabies: long-term induction of defense gene expression in potato, 926

Strømeng, G. M., S3.115, S3.116

Struck, C., 532

Studholme, D. J., S3.102

Stumpf, E., S3.10

Su, C. C., S3.115

Su, Y. H., S3.69

Suarez, S. N., S3.115

Subbarao, K. V., 282, 564, 641, 779, 908, 1349, S3.53, S3.61, S3.180 
Subbotin, S., S3.117

Subedi, A., S3.10

Subedi, N., S3.115

Subramaniam, R., S3.123

Subramanian, S., S3.116

Succinate dehydrogenase-inhibiting (SDHI) fungicide resistance: in Alternaria solani, molecular characterization, 40; in Botrytis cinerea, 347

Sugano, J., S3.79, S3.162

Sugio, A., S3.150

Sule, S., 453

Sultanova, N., S3.36

Sumampong, G., S3.106

Summers, C. F., S3.115

Sun, J., S3.129

Sun, M.-H., 1314

Sun, Q., S3.71, S3.152

Sun, X., S3.131

Sundaraj, S., 202

Sundin, G., S3.175

Sundin, G. W., S3.3, S3.23, S3.111, S3.134

Suslow, K., S3.105

Suslow, T. V., S3.142, S3.150

Sutton, W., S3.153

Sutula, M., S3.5

Swett, C. L., S3.116

Synchytrium spp.: phylogeny, 422

-S. endobioticum: TaqMan PCR assay for detection in soil, 422

Synthetic antimicrobial peptides: postinfection activity against Stemphylium vesicarium in pear, 1192

Szabo, L. J., S3.56

Szostek, S., S3.116

Szurek, B., 520

T2 toxin: accumulation in field oats, 57

Tabima, J. F., S3.57

Tag, A. G., S3.179

Takacs, E., S3.152

Takada, J., S3.108, S3.119

Talgø, V., S3.115, S3.116

Tanaka, F. A. O., 804

Tanaka, T., S3.85

Tancos, M. A., S3.149

Tande, C., S3.3, S3.116

Tang, J., S3.140

Tanger, P., S3.171

Tanguay, P., S1.6, S3.83

TaqMan PCR assays: for Synchytrium

endobioticum in soil, 422

TaqMan-qPCR assays: for Cercospora kikuchii in soybean leaves, 1118

Tatalovic, N., S3.116

Tatineni, S., S3.94, S3.152, S3.177

Tayeh, C., 293

Taylor, C. G., S3.124

Taylor, P. W., S3.24

Taylor, P. W. J., S3.13, S3.32

Teale, S. A., S1.2, S3.158

TeBeest, D. O., S2.5, S2.9

Techniques: coupling spore traps and qPCR assays to detect downy mildews of spinach and beet, 1349; detection of Xanthomonas oryzae pv. oryzae and $X$. oryzae pv. oryzicola with a padlock probe-based assay, 1130; high-throughput automated image analysis to measure quantitative virulence of Zymoseptoria tritici, 985; novel diagnostic method for citrus stubborn disease, 188; real-time PCR assay for Aphanomyces euteiches, 1138; screening metagenomic data for viruses using e-probe diagnostic nucelic acid assay, 1125; TaqMan PCR assay for
Synchytrium endobioticum, 422; TaqMan-qPCR assay for Cercospora kikuchii in soybean leaves, 1118; Verticillium dahliae race 2 -specific PCR, 779

Teichmann, B., S1.3

Tekete, C., 520

Temple, K., S3.50

Temple, T. N., S3.56

Teng, P. S., S3.150

Tenuta, A., S3.58, S3.132

Tenuta, M., S3.49, S3.81, S3.117

Teper-Bamnolker, P., 240

Teplier, R., 1360

Teresani, G. R., 804

Tesfaendrias, M., S3.117

Testen, A. L., 379, S3.156

Thammina, C., S3.146

Thapa, N., S2.10, S3.117

Thebaud, G., S3.98

Thekke-Veetil, T., S3.117, S3.141

Theobroma cacao: frosty pod rot, effects of shade tree spatial structure on, 275

Thevetia peruviana: leaf extracts, efficacy against wheat leaf rust, 933

Thielaviopsis basicola: effects on cotton growth and root morphology, 507

Thies, J. A., S3.117

Thiessen, L., S3.118

Thinakaran, J., S3.64

Thines, M., 1349, S3.61

Thomas, A., S3.118

Thomas, K. M., S3.161

Thomas, T. L., S3.179

Thomashow, L. S., 248

Thomas-Sharma, S., S3.51, S3.118

Thompkins, A. N., S3.52

Thompson, B. D., S3.180

Thompson, J. R., 211, 1232, S3.63

Thomsen, I. M., S3.116

Thrips tabaci: transmission of center rot of onion, 812

Thurber, C. S., S3.24

Tian, P., S3.118

Tian, T., 1314

Tian, Y., 1130

Tiedje, J., S3.159

Timilsina, S., S2.10, S3.119

Timper, P., S3.30, S3.92

Tipton, J., S3.39

Tisserat, N., S3.35, S3.148

Tisserat, N. A., S3.53, S3.135

Todd, J. C., 1360

Todd, T., S3.173

Todd, T. C., S3.171

Tohamy, A. M. M., S3.37

Tohme, J., 436

Tolbert, A. C., S3.113

Tomaso-Peterson, M., S2.9, S2.11, S3.2, S3.19, S3.113, S3.119, S3.122

Tomato spotted wilt virus resistance: in peanut, impact of, 202

Tonnessen, B., S3.119

Tonos, J., S3.135

Toomajian, C., S3.173

Toporek, S. M., S3.176

Torres, R., S3.92

Torres-Barragan, A., S3.129

Torres-Londono, G. A., S3.176

Tosa, Y., 634

Townes, A. L., S3.176

Toyoda, K., S3.108, S3.119

Tran, T., S3.119

Tran, T. M., S3.177

Tran, T. T., S3.119

Trandem, N., S3.86
Transcriptional activator-like effector (TALE) genes: genetic diveristy in Xanthomonas oryzae pv. oryzae, China, 672

Traore, S. M., 1152

Trapero, C., S3.120

Travadon, R., S3.12, S3.43, S3.67

Trehalose: induction of wheat defenses to powdery mildew, 293

Tremblay, D. M., S3.22

Trichoderma harzianum T39: gray mold resistance induced in tomato, 150

Tricothecene: fitness attributes of Fusarium graminearum isolates with 3-ADON or 15ADON genotypes, 513; production by Fusarium graminearum related to aggressivenss, 357

Trifloxystrobin resistance: in Venturia inaequalis, heteroplasmy of the $c y t b$ gene and, 945

Trigiano, R. N., S3.48

Trinsoutrot-Gattin, I., 1138

Tripathi, D., S3.120

Triplett, E. W., S3.155

Triplett, L., 436, 520, S3.120

Trippe, A., S3.87

Triticum spp.

-T. aestivum: biological control of root diseases

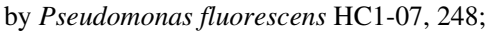
effects of Pyricularia oryzae on leaf gas exchange and chlorophyll $a$ fluorescence in silicon inoculated plants, 143; Fusariumdamaged kernels and deoxynivalenol in Fusarium-infected winter wheat, 472; Fusarium graminearum toxigenic capacity and trichothecene production related to aggressiveness in, 357; high-throughput automated image analysis to measure quantitative virulence of Zymoseptoria tritici, 985 ; identification of a hidden resistance gene in tetraploid wheat, 634; leaf rust resistance in breeding lines, molecular mapping, 865; pathogenesis-related protein expression in leaves pre-treated against leaf rust with plant extracts, 933; Pratylenchus thornei resistance, characterization of, 174; Puccinia striiformis resistance, race specificity, 1042; Puccinia striiformis resistance, Yr36 gene and, 871; Pyricularia oryzae impairment of leaf photosynthesis, 34; trehalose induction of defenses to powdery mildew, 293

$-T$. dicoccoides: identification of a hidden resistance gene in, 634

-T. turgidum: genetic analysis of leaf rust resistance, 1322

Trkulja, N. R., S3.34, S3.120

Tronsmo, A. M., 954

Truchon, A. N., S3.120, S3.168, S3.177

Trueman, C., S3.117

Truke, M. J., S3.7

Tsai, Y. C., S2.2, S2.10

Tseng, T. M., S3.177

Tsilo, T. J., 865

Tsuda, S., 964

Tubana, E. B. S., S2.2

Tucker, J. R., S3.120

Turcotte, R. M., S3.148

Turechek, W., S3.45, S3.127

Turgeman, T., 240

Turkington, T. K., S3.138

Turnbull, G. D., S3.42, S3.53, S3.138

Turner, M. F., S3.171

Tweddell, R., S3.63

Twizeyimana, M., S3.182

Twomey, M., S3.45, S3.149

Tychon, B., S3.37

Tyler, B., S3.133

Tylka, G, S3.177 
Tylka, G., S3.116

Tymon, L., S3.158

Type III secretion effector genes: Acidovorax citrulli lineages and, 1152

Tzanetakis, I. E., S3.32, S3.52, S3.106, S3.117

Uauy, C., 871

Uchida, J., S3.79

Uddin, M. N., S3.156

Uddin, W., 614

Ullman, D. E., 812

Ulloa, M., S3.126

Urashima, A., S3.6, S3.121

Uribe-Cortés, B., S3.18

Urrea, K. E., S2.10, S2.11, S3.121

Uyeda, J., S3.162

Uyoh, E. A., S3.35

Vaiciunas, J., S1.6

Valent, B., S3.125

Valenzuela-Solano, C., S3.179

Valkonen, J. P., S3.151

Vallad, G. E., S2.5, S2.10, S3.119

Vallejos, C., S3.93

Valverde, R. A., S3.59

van den Berg, F., 1264

van den Bosch, F., 1264

van der Burgt, A., S3.154

van der Graaff, E., 1283

Van der Heyden, H., 604, S3.65

van de Vossenberg, B. T., S3.121

Van Deynze, A. E., 479

Van Dorsselaer, A., 1021

Van Dyk, D. J., S3.121

van Heusden, S. W., S3.39

van Leeuwen, G. C., S3.121

van Rijswick, P. C., S3.121

Vargas, J. M., S3.143

Varvaro, L., 387

Vásquez-Céspedes, E., S3.82

Vaz Jauri, P., S3.6

Vazquez, O., S3.121

Vega, B., S3.121

Vegetative compatibility groups: complex mo-

lecular relationship in Verticillium dahliae, 650

Veloukas, T., 347

Venkateshwaran, M., S3.122

Venturi, V., 224, S3.28, S3.155

Venturia inaequalis: heteroplasmy of the

cytochrome $b$ gene and its role in trifloxystrobin resistance, 945

Vera, D. I., S3.122, S3.185

Verchot, J., S2.2

Verdier, V., 520, S3.120

Verhalen, L. M., 1088

Verreet, J. A., S3.61, S3.122

Verticillium spp.: systematics and evolution, review, 564

-V. dahliae: clonal expansion in lettuce, 641 ; on lettuce, model for multiseasonal spread of, 908 ; molecular relationship between vegetative compatibility groups in, 650; race 2 strains in spinach seed lots, 779

$-V$. nonalfalfae: pathogenicity and virulence of isolates from Ailanthus altissima, 282, cover photo: March

Verticillium wilt management: improving species identification and, 564

Viana de Sousa, M., S3.37

Vicré-Gibouin, M., 1138

Victoria, J. I., S3.3, S3.21

Vidalakis, G., 188

Viera, J. P., S3.91

Vigné, F., 586

Vike, E., S3.115

Villalobos, W., S3.82

Villani, S., S3.13, S3.29, S3.83
Villani, S. M., 945, S3.122

Villar, A., S3.6

Villari, C., S3.107

Vincent, D., 293

Vines, P. L., S2.11, S3.122

Vinokur, Y., 240

Virology: Alfalfa mosaic virus in Spain, variability and genetic structure, 1241; Bean common mosaic virus, interaction of genetic determinants with common bean resistance factors, 786; Bean common mosaic virusinduced temperature-insensitive necrosis in common bean, 1251; Cucumber mosaic virus, molecular genetic analysis of populations infecting pepper in Korea, 993; Fig mosaic virus, genetic variation and evolution, 108; Grapevine red blotch-associated virus in the U.S., 1232; Grapevine vein clearing virus, genetic diversity and tissue and host specificity, 539; grapevine viral infections, profiling using a randomly primed RT-PCR/macroarray multiplex platform, 211; Grapevine virus A, genetic diversity, Washington and California, 548; Plum bark necrosis stem pitting-associated virus, characterization and development of a broad-spectrum detection assay, 660; pollen transmission of Asparagus virus 2 and mixed infections in asparagus plants, 1001; screening metagenomic data for with the e-probe diagnostic nucleic acid assay, 1125; Southern rice black-streaked dwarf virus and Rice ragged stunt virus co-infection, 196, 794; Tomato spotted wilt virus resistance in peanut, impact of, 202; viruses in maize and Johnsongrass in southern Ohio, 1360; Wheat dwarf virus, localization and distribution in leafhopper vector, 897; Wheat yellow mosaic virus, biological and genetic diversity, 313

Visser, R. G., S3.39

Vitis spp.: geographic distribution of cryptic species of Plasmopara viticola in, 692

$-V$. riparia: downy mildew on phylloxera galls of, cover photo: July

$-V$. vinifera: differential responses of cultivars to Botryosphaeria dieback, 1021; viral infections profiled with a randomly primed RT-PCR/ macroarray multiplex platform, 211

Vito, L. M., S3.30, S3.48

Vittal, R., 86

Vogel, J., S3.40

Vogel, K., S3.114, S3.134

Voglmayr, H., 1349, S3.61

Vojnov, A. A., 970

von Bodman, S., S3.130

Vorholt, J. A., S3.155

Vorsa, N., S3.129

Voshell, R. J., S3.163

Voth, W. D., S3.120

Voytas, D. F., S3.141

Vrient, A., S3.136

Vrisman, C. M., S3.123

Vy, T. T. P., 634

Wadl, P. A., S3.30, S3.48

Waisen, P., S3.123

Walcott, R., 1152

Walcott, R. R., 1130

Walenta, D. L., S3.181

Walia, J. J., 108

Walker, D., S3.69

Walker, N., S2.8

Walker, N. R., S2.4, S3.44

Walkowiak, S., S3.123

Wallace, E. C., S3.123

Waller, T. J., S1.6, S3.123, S3.164

Wallhead, M., S3.46, S3.123, S3.124

Wallingford, A. K., 126
Wallington, E., 871

Wallis, C., S3.27, S3.124

Wallis, C. M., 126

Walsh, E., S3.124

Walter, G., S3.51

Walter, M., 50

Wamishe, Y., S2.11, S3.124

Wamishe, Y. A., S3.125

Wan, A., S3.125

Wang, A., S3.25, S3.135, S3.152

Wang, C., S3.37, S3.55, S3.126

Wang, D., S3.125, S3.182

Wang, D. H., S3.182

Wang, H., 196, 794, S3.73, S3.127

Wang, J., S3.40, S3.63, S3.126, S3.127, S3.134

Wang, J. F., S3.156

Wang, L., S3.89, S3.123

Wang, M., S3.39, S3.125

Wang, M. L., S3.32

Wang, N., 134, S3.8, S3.41, S3.69, S3.135, S3.137

Wang, N. Y., S3.126

Wang, S., S3.17, S3.125

Wang, W., S3.126

Wang, X., 897, S3.25, S3.73, S3.74, S3.119, S3.125

Wang, X. W., S3.125

Wang, Y., 897, S3.49

Wang, Y. T., 445

Wang, Z., 269, S3.126

Wanner, L. A., S3.127

Warburton, M. L., S3.140

Ward, B. M., S2.2, S3.109

Ward, N. A., 1118

Ward, T. J., S3.140

Warmington, R., S3.86

Warner, A., S3.127

Warren, J., S3.127

Washington-Keizerweerd, A., S3.128

Wass, B., S3.128

Watrin, C., S3.86

Weaver, B. D., S3.68

Weaver, M. A., S3.128

Webb, K. M., 886, S3.128

Webb, M. A., S2.2

Webster, T., S2.2

Wechter, W. P., S3.128

Weems, J. D., S3.129

Weerasooriya, D. K., S3.11

Wei, G. W., S3.92

Wei, Q., S3.95

Wei, T., 897

Wei, W., S3.161

Wei, Y., S3.137

Weiland, J., S2.4, S3.129

Weiland, J. E., S3.44

Weimer, J., S1.4, S3.83

Weis, K. L., S2.11

Welbaum, G., 1152

Weller, D. M., 248

Wells, L. D., S3.129

Welty, C., S3.12

Wen, S.-S., 248

Wen, X., 158

Wenninger, E. J., S3.85, S3.180

Were, V., 1221

Weselowski, B., S3.1

West, J. L., S1.2

Westenberg, M., S3.121

Westerdahl, B. B., S3.129

Westerhof, J., S3.78

Westerveld, S., S3.28

Wettstein, D., von, 248

Wheat dwarf virus: localization and distribution in leafhopper vector, 897

Wheat yellow mosaic virus: biological and genetic diversity, 313 
Wheeler, D. L., S3.185

Wheeler, T., S3.131

Whitaker, V. M., 67

White, F., S3.178

White, F. F., S2.10

White, J. R., S3.87

Whitfield, A., S3.177

Whitfield, A. E., S3.11, S3.158

Whittington, H. D., S3.129

Wicker, E., 586

Wickramasinghe, D., S3.165, S3.166

Widmer, T. L., S3.105

Wiechel, T., S3.32

Wiersma, J. J., S3.8

Wiggins, G. J., S3.30, S3.48

Wijeratne, A., 1360

Wijeratne, S., 1360

Wildolm, J. M., 843

Wilkerson, T. H., S3.129

Willbur, J. F., S3.130, S3.171

Willemsen, A., 108

Williams, J., S3.21

Williams, K. L., S3.130

Williams, K. M., S3.130

Williams, S. D., S3.68

Williams, W. P., S3.140

Williford, L., S3.130

Willis, R., S2.10

Willocquet, L., S3.104

Wilson, J., S3.167

Wilson, L., S3.82

Wilson, N. L., 933

Wilson, R. A., S3.74, S3.170

Windham, G., S3.140

Windham, M. T., S3.30, S3.48

Wintermantel, W. M., S3.128, S3.130

Wise, A., 692

Wise, C., 1036

Wise, J. C., S3.3

Wise, K., S3.173

Wise, K. A., S3.58, S3.170

Wisotsky, S. R., 15

Withers, S., S3.130

Woeste, K. E., S3.54, S3.135

Wolf, D., S3.144

Wolfenbarger, S., S3.45, S3.149

Wonni, I., 520

Wood, B. W., S3.15, S3.16

Wood, D., S3.148

Woodell, L. K., S3.104

Wood rot of citrus: characterization of

basidiomycetes associated with, Italy, 851

Woodward, J., S2.5

Woodward, J. E., S2.11, S3.131

Workneh, F., 126, S3.88, S3.109, S3.131, S3.158

Worthington, C. J., 75

Wosula, E. N., S3.131

Wrather, J. A., S3.38

Wu, B. M., 908, S3.136

Wu, J., S3.56, S3.131

Wu, S., S3.158, S3.164

Wu, W., S3.89

Wu, X., S3.137

Wunsch, M., S3.52

Wyenandt, C. A., S1.4

Xanthomonas spp.

-X. arboricola pv. juglandis: colonization of dormant walnut buds, 1163

-X. axonopodis pv. malvacearum resistance: in cotton, pyramiding $B$ genes and, 1088

-X. citri subsp. citri: control with foliar application of biofilm formation-inhibiting compounds, 134
-X. citri subsp. citri resistance: in Satusma mandarin 'Okitsu', role of leaf features in, 970

-X. euvesicatoria: role of blossom colonization in pepper seed infestation, 232

$-X$. oryzae pv. oryzae: detection in rice with a padlock probe-based assay, 1130; genetic diversity of transcriptional activator-like effector genes, China, 672

$-X$. oryzae pv. oryzicola: detection in rice with a padlock probe-based assay, 1130; population analysis, Mali and Burkina Faso, 520

Xi, P., S3.131

Xian, M., S3.60

Xiang, N., S3.132

Xiao, C. L., S3.4, S3.101, S3.132

Xiao, K., S3.41

Xiao, S., S3.25

Xie, F., S3.161

Xing, Y., S3.51

Xiong, L., 672

Xiong, Z., S3.132, S3.134

$\mathrm{Xu}, \mathrm{D} ., 196, \mathrm{~S} 3.137$

$\mathrm{Xu}, \mathrm{F} ., 224$

Xu, J. R., S3.48

$\mathrm{Xu}, \mathrm{M} ., 158$

Xu, M. L., 539

$\mathrm{Xu}, \mathrm{R} ., 1130$

Xu, S. S., S3.58, S3.71, S3.136

$\mathrm{Xu}, \mathrm{W} ., \mathrm{S} 3.140$

$\mathrm{Xu}, \mathrm{X} ., 57$

Xue, A., S3.79, S3.132

Xylanase: Fusarium graminearum disease severity in soybean, 1201

Xylella fastidiosa: blocked diffusible signal factor and insect transmission, 27; genetic structure in sweet orange, 120

Yaeno, T., S3.85

Yamada, T., S3.4, S3.143

Yamaoka, N., S3.85

Yang, A., S3.132

Yang, H., S3.178

Yang, J., S3.133

Yang, L., S3.132

Yang, M.-M., 248

Yang, X., S3.85, S3.133

Yang, X. B., S3.173

Yang, X. P., S3.11

Yang, Y., S3.25

Yasmin, T., S3.133

Ye, J. L., 445

Ye, W., S3.59

Yendrek, C. R., S3.24

Yessenbekova, G., S3.133

Ying, L., S3.57

Yokomi, R. K., S3.133

Yokota, K., S3.134

Yordem, B., S3.134

Yoshida, F., S3.72

You, F. M., S3.36

Youn, G., S3.57

Young, C. A., S3.116

Young, R. F., S3.140

Young-Kelly, H. M., S2.3, S2.5

Yr36 gene: wheat partial resistance to Puccinia striiformis and, 871

Yu, J. M., S3.125

Yu, K., S3.37, S3.69, S3.112

Yu, N., S3.134

Yu, Z., S3.136

Yuen, G., S3.134

Yuen, G. Y., S3.114

Yuen, J., S3.118
Yuen, J. M. F., S3.16

Yun, J. C., S3.60, S3.107

Zaccaron, M., S3.134

Zahir, U. M., S3.156

Zakria, M., 672

Zala, M., 95, S3.24

Zarka, D., S3.169

Zasada, I., S3.85

Zasada, I. A., S3.80

Zaworski, E., S3.177

Zea mays: mycotoxin contamination in Kenyan mills, 1221; viruses in maize and Johnsongrass in southern Ohio, 1360

Zebra chip disease. See 'Candidatus Liberibacter solanacearum'

Zeng, Q., S3.3, S3.31, S3.134

Zerillo, M. M., S3.53, S3.135

Zernova, O. V., 843

Zhai, C., S3.89

Zhai, Y., S3.89

Zhang, B., S3.161

Zhang, C., S3.32, S3.135, S3.178

Zhang, D.-D., 472

Zhang, G., S3.135

Zhang, H., S3.39, S3.136

Zhang, J., S3.94, S3.174, S3.177, S3.178

Zhang, K., S3.126

Zhang, L., S3.1, S3.131, S3.135

Zhang, M., S3.137

Zhang, P., S3.132

Zhang, R., S3.136

Zhang, S., S3.41, S3.73, S3.90, S3.132, S3.136, S3.145

Zhang, X., S3.40, S3.126, S3.135, S3.136, S3.137

Zhang, Y., 539, S3.89, S3.134, S3.135

Zhang, Z., S3.55

Zhao, B., 1152, S3.158, S3.161, S3.164

Zhao, G., S3.48

Zhao, J., S3.126

Zhao, L., S3.32

Zhao, M., S3.136

Zhao, Y., 1130, S3.24, S3.67, S3.161

Zheng, J., 1289, S3.98

Zheng, Y. X., S3.136

Zheng, Z., S3.27, S3.137

Zhong, S., S3.4, S3.67, S3.108, S3.136

Zhou, C., S3.158, S3.164

Zhou, G., 196, 794, S3.137

Zhou, H., S3.137

Zhou, P., S3.134

Zhou, Q., S3.53

Zhou, X., S3.48, S3.137

Zhou, X. G., S3.82, S3.137, S3.138

Zhou, Y., S3.96

Zhu, S., S3.69

Zhu, W., 445

Zhu, X., S3.138

Zhuang, X., S3.40

Ziegler-Graff, V., S3.81

Ziesman, B. R., S3.138

Zitnick-Anderson, K. K., S3.138

Živanović, M., 1152

Zlesak, D. C., S3.87

Zoffoli, J. P., S3.33, S3.40

Zou, L.-F., 672

Zouhar, M., 306

Zucchi, T. D., 1298

Zuniga, A., S3.8

Zuñiga-Mendoza, E., S3.24

Zuzak, K. A., S3.138

Zymoseptoria tritici: measurement of quantitative virulence with high-throughput automated image analysis, 985 